\title{
THE DETERMINED PROPERTY OF BAIRE IN REVERSE MATH
}

\author{
ERIC P. ASTOR, DAMIR DZHAFAROV, ANTONIO MONTALBÁN, REED SOLOMON, \\ AND LINDA BROWN WESTRICK
}

\begin{abstract}
We define the notion of a completely determined Borel code in reverse mathematics, and consider the principle CD-PB, which states that every completely determined Borel set has the property of Baire. We show that this principle is strictly weaker than $\mathrm{ATR}_{0}$. Any $\omega$-model of CD-PB must be closed under hyperarithmetic reduction, but CD-PB is not a theory of hyperarithmetic analysis. We show that whenever $M \subseteq 2^{\omega}$ is the second-order part of an $\omega$-model of CD-PB, then for every $Z \in M$, there is a $G \in M$ such that $G$ is $\Delta_{1}^{1}$-generic relative to $Z$.
\end{abstract}

\section{INTRODUCTION}

The program of reverse mathematics aims to quantify the strength of the various axioms and theorems of ordinary mathematics by assuming only a weak base theory $\left(\mathrm{RCA}_{0}\right)$ and then determining which axioms and theorems can prove which others over that weak base. Five robust systems emerged, (in order of strength, $\left.\mathrm{RCA}_{0}, \mathrm{WKL}_{0}, \mathrm{ACA}_{0}, \mathrm{ATR}_{0}, \Pi_{1}^{1}-\mathrm{CA}_{0}\right)$ with most theorems of ordinary mathematics being equivalent to one of these five (earning this group the moniker "the big five"). The standard reference is Sim09. In recent decades, most work in reverse mathematics has focused on the theorems that do not belong to the big five but are in the vicinity of $\mathrm{ACA}_{0}$. Here we discuss two principles which are outside of the big five and located in the general vicinity of $\mathrm{ATR}_{0}$ : the property of Baire for completely determined Borel sets (CD-PB) and the Borel dual Ramsey theorem for 3 partitions and $\ell$ colors (Borel-DRT ${ }_{\ell}^{3}$ ). Both principles involve Borel sets.

Our motivation is to make it possible to give a meaningful reverse mathematics analysis of theorems whose statements involve Borel sets. The way that Borel sets are usually defined in reverse mathematics forces many theorems that even mention

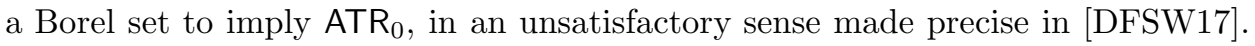
Here we propose another definition for a Borel set in reverse mathematics, distinguished from the original by the terminology completely determined Borel set, and to put bounds on the strength of the statement

CD-PB : "Every completely determined Borel set has the property of Baire"

This statement should be compared with the usual "Every Borel set has the property of Baire", which [DFSW17] showed is equivalent to $\mathrm{ATR}_{0}$ for aforementioned empty reasons. In contrast, working with CD-PB requires working with hyperarithmetic generics, giving this theorem more thematic content. While we do not claim

Dzhafarov was supported by grant DMS-1400267 from the National Science Foundation of the United States and a Collaboration Grant for Mathematicians from the Simons Foundation. 


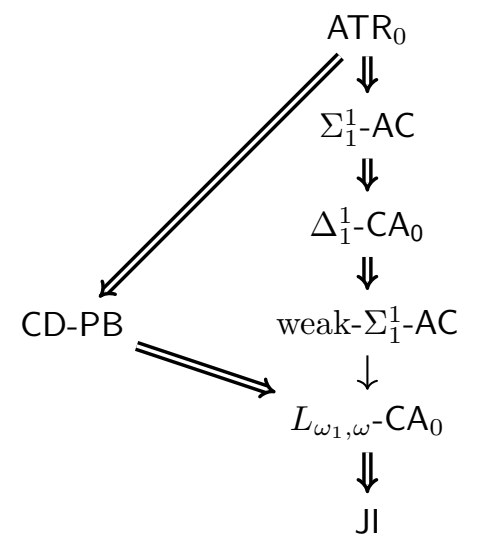

Figure 1. CD-PB, ATR , $_{0}$ and some theories of hyperarithmetic analysis. The new results are those concerning CD-PB. A double arrow indicates a strict implication.

that CD-PB is the "right" formalization of the principle that Borel sets have the Baire property, it is a step in that direction.

We show that over RCA, CD-PB is implied by ATR $R_{0}$ and implies $\mathrm{L}_{\omega_{1}, \omega}-C A$. Our first main theorems say that both implications are strict.

Theorem 1.1. There is an $\omega$-model of $\mathrm{CD}-\mathrm{PB}$ in which $\mathrm{ATR}_{0}$ fails.

Theorem 1.2. There is an $\omega$-model of $\mathrm{L}_{\omega_{1}, \omega}$-CA in which CD-PB fails. In fact, $H Y P$ is such an $\omega$-model.

This establishes that CD-PB is located in the general vicinity of the theories of hyperarithmetic analysis, a mostly linearly ordered collection of logical principles which are strong enough to support hyperarithmetic reduction, but too weak to imply the existence of jump hierarchies. With the exception of Jullien's indecomposability theorem [Mon06, no theorems of ordinary mathematics are known to exist in this space. The only known statement of hyperarithmetic analysis that is not linearly ordered with the others is the arithmetic Bolzano-Weierstrass theorem (see [Fri75], Con12]). Now, CD-PB is not a theory of hyperarithmetic analysis because it does not hold in $H Y P$. However these theories of hyperarithmetic analysis are the closest principles to CD-PB that have already been studied.

To elaborate on the factors preventing to CD-PB from being a theory of hyperarithmetic analysis, we prove the following generalization of Theorem 1.2 above, establishing that hyperarithmetic generics must appear in any $\omega$-model of CD-PB.

Theorem 1.3. If $\mathcal{M}$ is an $\omega$-model of $\mathrm{CD}-\mathrm{PB}$, then for any $Z \in M$, there is a $G \in M$ that is $\Delta_{1}^{1}(Z)$-generic.

As an application, we use CD-PB to analyze the theorem Borel-DRT ${ }_{\ell}^{3}$, whose statement contains no concept of mathematical logic apart from that of Borel sets. (The statement of this theorem can be found in Section 8.) We show that, under appropriate formalization, Borel-DRT ${ }_{\ell}^{3}$ is strictly weaker than $\mathrm{ATR}_{0}$ and shares some 
properties with the theories of hyperarithmetic analysis. It is left open whether Borel-DRT $T_{\ell}^{3}$ is a statement of hyperarithmetic analysis.

Theorem 1.4. For any finite $\ell \geq 2$, the principle Borel-DRT $\mathrm{DT}_{\ell}^{3}$ is strictly implied by $\mathrm{ATR}_{0}$. Any $\omega$-model of Borel-DRT ${ }_{\ell}^{3}$ is closed under hyperarithmetic reduction.

The first section gives the preliminaries. In Section 2 we give the definition of a completely determined Borel code and prove its basic properties. In Section 3 we

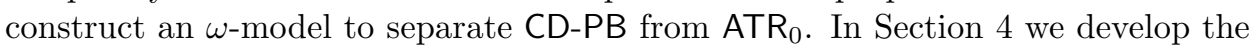
machinery of decorating trees which will be used in Sections 5 and 6. In Section 5 , we prove that CD-PB does not hold in $H Y P$. In Section 6 , we prove Theorem 1.3. This is a strictly stronger theorem than the one proved in Section 5 , but also a bit longer to prove, so Section 5 could be regarded as a warm-up. In Section 7 we give an application to the Borel dual Ramsey theorem. Section 8 contains open questions.

The authors would like to thank Julia Knight and Jindra Zapletal for helpful discussions on this topic, and the anonymous referee for many suggestions which have made the arguments clearer and more accessible.

\section{Preliminaries}

2.1. Notation, Borel sets and Borel codes. We typically denote elements of $\omega^{<\omega}$ by $\sigma, \tau$ and elements of $2^{<\omega}$ by $p, q$. We write $\sigma \preceq \tau$ to indicate that $\sigma$ is an initial segment of $\tau$, with $\prec$ if $\sigma \neq \tau$. We may also use this notation to indicate when a finite string is an initial segment of an infinite string. For $p \in 2^{<\omega}$, the notation $[p]$ refers to the set $\left\{X \in 2^{\omega}: p \prec X\right\}$. The empty string is denoted by $\lambda$. A string with a single component of value $n \in \omega$ is denoted by $\langle n\rangle$. String concatenation is denoted by $\sigma^{\frown} \tau$. Usually we write $\sigma^{\frown} n$ instead of the more technically correct but uglier $\sigma^{\curvearrowright}\langle n\rangle$.

If $U$ is a set of strings (for example, a tree, or a coded open subset of $2^{\omega}$ ), and $\sigma$ is any string, we write $\sigma^{\wedge} U$ to mean $\left\{\sigma^{\wedge} \tau: \tau \in U\right\}$. If $T$ is a tree and $\sigma \in T$, we write $T_{\sigma}$ to mean $\left\{\tau: \sigma^{\wedge} \tau \in T\right\}$.

The Borel subsets of a topological space are the smallest collection which contains the open sets and is closed under complements and countable unions (and thus countable intersections).

A Borel code is a well-founded tree $T \subseteq \omega^{<\omega}$ whose leaves are labeled by basic open sets or their complements, and whose inner nodes are labeled by $\cup$ or $\cap$. The Borel set associated to a Borel code is defined by induction, interpreting the labels in the obvious way. Any Borel set can be represented this way, by applying DeMorgan's laws to push any complementation out to the leaves.

We use standard recursion-theoretic notation. The eth Turing functional is denoted $\Phi_{e}$. A pair of natural numbers $(n, m)$ is coded as a single natural number $\langle n, m\rangle$ via a canonical computable bijection between $\mathbb{N}$ and $\mathbb{N} \times \mathbb{N}$. Although this notation $\langle n, m\rangle$ could also refer to a string with two elements, context will make it clear which type is meant.

2.2. Reverse mathematics. We assume the reader is familiar with the program of reverse mathematics. The standard reference on this subject is Sim09. Here we just recall that the principle of arithmetic transfinite recursion is formulated as follows. If $X \in 2^{\omega}$ codes a linear order on some subset of $\mathbb{N}$, let $<_{X}$ denote that linear order and (abusing notation) let $X$ also denote the domain of the linear 
order. Assuming there is a linear order $X$ in the context, given $Y \in 2^{\omega}$ and $a \in X$, we let $Y^{a}$ denote $\left\{\langle n, b\rangle \in Y: b<_{X} a\right\}$. Given an arithmetic predicate $\phi(n, Z)$, we define the predicate $H_{\phi}(X, Y)$ as follows:

$$
H_{\phi}(X, Y) \equiv \quad X \text { is a linear order and } Y=\left\{\langle n, a\rangle: a \in X \text { and } \phi\left(n, Y^{a}\right)\right\} .
$$

The principle $\mathrm{ATR}_{0}$ is a scheme ranging over arithmetic formulas $\phi$, which states that for each such $\phi$, if $X$ is a well-order, then there is a $Y$ such that $H_{\phi}(X, Y)$. Using $\mathrm{ACA}_{0}$, one can show that such $Y$ is unique. For details, see Sim09, Section V.2].

In the special case where $\phi(n, Z)$ is the jump operator, that is $\phi(n, Z) \equiv n \in Z^{\prime}$, then any $Y$ satisfying $H_{\phi}(X, Y)$ is called a jump hierarchy on $X$.

The principle of effective transfinite recursion is defined almost the same as $\mathrm{ATR}_{0}$, but using $\Delta_{1}^{0}$ formulas instead of arithmetical formulas. In [DFSW17] it is shown that effective transfinite recursion also goes through in $\mathrm{ACA}_{0}$.

Both $\mathrm{ATR}_{0}$ and effective transfinite recursion are used to define objects by recursion along a well-order $X$. If we only want to use induction to verify some arithmetic property of a family of objects indexed by $X$, the principle of arithmetic transfinite induction is used and this principle also holds in ACA $_{0}$ (Sim09, Lemma V.2.1]).

In reverse mathematics, the role of an ordinal is played simply by a well-founded linear order. For certain of our constructions it is convenient to have a more structured well-order for which the operation of finding a successor is effective. For that reason we also use the terminology of Kleene's $\mathcal{O}$, which is briefly reviewed in the next section.

2.3. Ordinal notations and pseudo-ordinals. We assume the reader is familiar with ordinal notations and pseudo-ordinals. A standard reference is Sac90. Here we give just a brief summary of the concepts and techniques that we use. Recall that Kleene's $O$, denoted $\mathcal{O}$, is a $\Pi_{1}^{1}$-complete subset of $\omega$ consisting of notations for all the computable ordinals, where 1 denotes the ordinal $0,2^{a}$ denotes the successor of the ordinal denoted by $a$, and $3 \cdot 5^{e}$ denotes the limit of the ordinals denoted by $\Phi_{e}(n)$, provided that $\Phi_{e}$ is total and for all $n, \Phi_{e}(n)<_{*} \Phi_{e}(n+1)$ (where $<_{*}$ is the transitive closure of the relation defined by $1<_{*} x$ if $x \neq 1, x<_{*} 2^{x}$, and $\left.\Phi_{e}(n)<_{*} 3 \cdot 5^{e}\right)$. The notation $\leq_{\mathcal{O}}$ refers to the relation $<_{*}$ restricted to $\mathcal{O}$.

To avoid excessive repetition of the phrase "denoted by", henceforth we will conflate ordinals with their notations. A given ordinal may have many notations, but for each such notation $a,\left\{b \in \mathcal{O}: b<_{\mathcal{O}} a\right\}$ is linearly ordered by $\leq_{\mathcal{O}}$, so canonical names for the ordinals below $a$ are implied by the choice of $a$. We will also write $a+k$ for the $k$ th successor of $a$ (instead of the technically accurate but more cumbersome tower of exponentials), and $a-k$ for its $k$ th predecessor when this exists. Although $a$ is technically an element of $\omega$, it would never make sense to add or subtract an ordinal using the usual addition on the natural numbers, so this should not create confusion. Also, sometimes we will take a fixed but unspecified number of successors of $a$, and the result is denoted $a+O(1)$.

The set $\left\{b \in \mathcal{O}: b<_{\mathcal{O}} a\right\}$ is c.e. uniformly in $a$, because the relation $<_{*}$ is a c.e. relation. Throughout, we let $p$ denote the computable function such that for each $a \in \omega$, we have $W_{p(a)}=\left\{b \in \omega: b<_{*} a\right\}$.

The definition of $\mathcal{O}$ also relativizes to any oracle $X$, producing a $\Pi_{1}^{1}(X)$-complete set $\mathcal{O}^{X}$ with the same properties as above. 
The basic tool for working with ordinal notations is effective transfinite recursion, which suffices to define a large swath of important constructions involving ordinal notations. These constructions also relativize (in reverse mathematics this corresponds to allowing a real parameter appear in the formula $\phi$ ). Here are two examples which are used in this paper. (Ranked formulas of $L_{\omega_{1}, \omega}$ are defined in the next subsections).

Proposition 2.1. Given an oracle $X$, an ordinal $a \in \mathcal{O}^{X}$, and a number $x \in \omega$, there is an a-ranked formula of $L_{\omega_{1}, \omega}$ which holds exactly if $x \in H_{a}^{X}$, where $H_{a}^{X}$ denotes the unique jump hierarchy (relative to $X$ ) on the well-order $W_{p(a)}^{X}$.

Proof. The existence of a formula of $L_{\omega_{1}, \omega}$ defining membership in $H_{a}^{X}$ follows directly from effective transfinite recursion applied to the definition of $H_{a}^{X}$. The fact that the formula can be $a$-ranked uses the normal form theorem for simplifying expressions involving bounded quantifiers. These simplifications can be carried out effectively.

Proposition 2.2. Given an $X$-computable linear order $L$, there is a number a such that $L$ is well-founded if and only if $a \in \mathcal{O}^{X}$. Furthermore, if $L$ is ill-founded, any descending sequence in $W_{p(a)}^{X}$ uniformly computes a descending sequence in $L$.

Proof. The first statement above is a special case of [Sac90, Lemma 4.3]. The second statement is true for the construction in [Sac90, but not explicitly stated there. So for the reader's convenience here is an alternative construction which establishes both parts of the proposition.

Let $L^{\prime}$ denote the linear order with order type $1+L+1$. Without loss of generality, the least element of $L^{\prime}$ is 0 and the greatest element of $L^{\prime}$ is 1 . Define a function $e: L^{\prime} \rightarrow \omega$ as follows. Let $e(0)=1$ (the latter being the code for ordinal $0)$. For $k \in L^{\prime}$ and $n \in \omega$, let $h(k, n)$ denote the $<_{L^{\prime}}$-greatest element among $\left\{j \leq n: j<_{L^{\prime}} k\right\}$. Then for $k \in L^{\prime}$ with $k \neq 0$, define $e(k)$ by effective transfinite recursion as follows.

$$
\Phi_{e(k)}^{X}(n)= \begin{cases}n \text { (i.e. the } n \text {th successor of } 0) & \text { if } h(k, n)=0 \\ 3 \cdot 5^{e(h(k, n))}+n & \text { otherwise. }\end{cases}
$$

Then let $a=e(1)$. It is routine to show that $e:\left(L,<_{L}\right) \rightarrow\left(W_{p(a)}^{X},<_{*}\right)$ is orderpreserving. Also, the order type of $W_{p(a)}^{X}$ is $\omega \cdot(1+L)$, with $e$ providing a selector for each $\omega$-chain in $W_{p(a)}^{X}$. If $L$ is well-founded, induction along $L$ shows that $a \in \mathcal{O}^{X}$. On the other hand, $e$ and its inverse (the inverse being applied to the $\omega$-chains of $\left.W_{p(a)}^{X}\right)$ provide an effective correspondence between any descending sequences in $L$ and in $W_{p(a)}^{X}$.

Kleene's $\mathcal{O}$ also has a $\Sigma_{1}^{1}$ superset $\mathcal{O}^{*}$, defined as the intersection of all $X \in H Y P$ such that $1 \in X, a \in X \Longrightarrow 2^{a} \in X$, and

$$
\forall n\left[\Phi_{e}(n) \in X \text { and } \Phi_{e}(n)<_{*} \Phi_{e}(n+1)\right] \Longrightarrow 3 \cdot 5^{e} \in X .
$$

Observe also that $\mathcal{O}$ is contained in $\mathcal{O}^{*}$ (the definition of $\mathcal{O}$ is the same, except that to get $\mathcal{O}$ we quantify over all $X$ rather than just those in $H Y P$ ). Then, since $O^{*}$ is $\Sigma_{1}^{1}$, it must be a proper extension of $\mathcal{O}$, and thus there must be elements in $a \in \mathcal{O}^{*} \backslash \mathcal{O}$. Such elements are called pseudo-ordinals. For all such $a, W_{p(a)}$ is an ill-founded linear ordering without hyperarithmetic descending sequences, and 
hence must be isomorphic to $\omega_{1}^{c k}+\omega_{1}^{c k} \cdot \mathbb{Q}+\beta$ for some computable ordinal $\beta$ (see Har68, Theorem 1.8]). In particular, for every pseudo-ordinal $a$ and every actual ordinal $\beta<\omega_{1}^{c k}$, there exists some $b<_{*} a$ which denotes $\beta$.

We will frequently use the following facts about pseudo-ordinals: any function on $\mathcal{O}$ defined by effective transfinite recursion with $H Y P$ parameters is also defined on all of $\mathcal{O}^{*}$, and any arithmetic properties of the resulting objects also hold for all of $\mathcal{O}^{*}$, provided those properties are proved by induction. These facts follow from the more general [Har68, Corollary 1.6], but they can also be easily seen in our reverse mathematics context, using the fact that effective transfinite recursion and arithmetic transfinite induction hold in $H Y P$, but $H Y P$ believes all pseudoordinals are ordinals.

2.4. Alternating and ranked trees. The following definition of a ranking for a tree is looser than given by some authors. We only require that the notations decrease, rather than the strong requirement that $\rho(\sigma)=\sup _{n}\left(\rho\left(\sigma^{\frown} n\right)+1\right)$. Additionally, it is technically convenient for us to assume that leaves have the smallest possible rank, but nothing serious hinges on this.

Definition 2.3. If $T \subseteq \omega^{<\omega}$ is any tree, and $\rho: T \rightarrow \mathcal{O}^{*}$, we say that $\rho$ ranks $T$ if

(1) for all $\sigma$ and $n$ such that $\sigma^{\frown} n \in T$, we have $\rho\left(\sigma^{\frown} n\right)<_{*} \rho(\sigma)$, and

(2) for each leaf $\sigma \in T, \rho(\sigma)=1$.

If $T$ is ranked by $\rho$ and $\rho(\lambda)=a$, we say that $T$ is a-ranked by $\rho$.

If $T$ is a ranked tree and the name of the ranking function is not explicitly given, then its name is $\rho_{T}$.

Trees appear for us in two contexts: as codes for formulas of $L_{\omega_{1}, \omega}$ and codes for Borel sets. In both cases, interior nodes are labeled with one of $\{\cap, \cup\}$. The nicest codes alternate these.

Definition 2.4. If $T \subseteq \omega^{<\omega}$ is a tree with a labeling function $\ell$ then we say $(T, \ell)$ alternates if for every $\sigma^{\urcorner} n \in T$, we have $\ell(\sigma) \neq \ell\left(\sigma^{\urcorner} n\right)$.

The main point about alternating trees is that it is always safe to assume that we have them. If we start with a labeled, $a$-ranked tree, we can effectively transform it into an alternating $a$-ranked tree, with no effect on the logic of the tree (assuming that whatever model we are working in does not contain any paths, if the tree is truly ill-founded.)

One small technical detail about this effective transformation will be used later, so we give the transformation explicitly. The definition is by effective transfinite recursion on the rank of the tree. Given an $a$-ranked tree $T$ with a $\cup$ at the root, define

$$
\operatorname{Alternate}(T)=\{\lambda\} \cup \bigcup_{\sigma \in A(T)}\left\langle n_{\sigma}\right\rangle^{\frown} \operatorname{Alternate}\left(T_{\sigma}\right),
$$

where $A(T)$ is the collection of all $\sigma \in T$ such that $\sigma$ is not a $\cup$, but each $\tau \prec \sigma$ is a $\cup$, and where $\sigma \mapsto n_{\sigma}$ is a computable injection from $\omega^{<\omega}$ to $\omega$; define the operation analogously when $T$ has a $\cap$ at the root, and of course the operation does nothing to a leaf. We see that this operation is just rearranging some subtrees by breaking them apart and reattaching them to a higher-ranking parent than their original attachment. The rank of any node in $\operatorname{Alternate}(T)$ is inherited from its rank in the original tree. Observe that every level-one subtree of the alternated tree (that is, every subtree of the form Alternate $(T)_{\left\langle n_{\sigma}\right\rangle}$ ) is the alternating version of a 
subtree of a single level-one subtree (namely $T_{\langle\sigma(0)\rangle}$ ) of the original tree. In other words, the process of making a tree alternate may break apart level-one subtrees, but never mixes them together.

2.5. Borel sets in reverse mathematics. In reverse mathematics, open subsets of $2^{\omega}$ are represented by sets of strings $p \in 2^{<\omega}$. If $U$ is such a code, we will abuse notation and write $X \in U$ to mean that for some $p \in U, p \prec X$. This is in addition to also sometimes speaking of the strings $p \in U$. Context will tell which usage is meant.

For arbitrary Borel sets, we will make a more careful distinction between code and object. We restrict attention to Borel subsets of $2^{\omega}$. A clopen subset $C$ of $2^{\omega}$ is represented by an element of $\omega$ which canonically codes a finite subset $F \subseteq 2^{<\omega}$. As above, for $X \in 2^{\omega}$, we say $X \in C$ if and only if $p \prec X$ for some $p \in F$. A code for $C$ as a clopen set gives more information about $C$ than an open code for the same set, because the number of elements of $F$ is computable from the code. Effectively in a standard code for a clopen set, one can find a standard code for its complement.

We take the following as the definition of a (labeled) Borel code in reverse mathematics.

Definition 2.5. A labeled Borel code is a well-founded tree $T \subseteq \omega^{<\omega}$, together with a function $\ell$ whose domain is $T$, such that if $\sigma$ is an interior node, $\ell(\sigma)$ is either $\cup$ or $\cap$, and if $\sigma$ is a leaf, $\ell(\sigma)$ is a standard code for a clopen subset of $2^{\omega}$.

We call this a labeled Borel code instead of a Borel code, because we have added a labeling function to the original definition to improve readability 11 If $\ell(\sigma)=\cup$ we may simply say " $\sigma$ is a union node", and similarly for $\cap$. We will also usually suppress mention of $\ell$, in an abuse of notation.

If $T$ is a labeled Borel code and $X \in 2^{\omega}$, the existence of an evaluation map is used to determine whether $X$ is in the set coded by $T$.

Definition 2.6. If $T$ is a labeled Borel code and $X \in 2^{\omega}$, an evaluation map for $X \in T$ is a function $f: T \rightarrow\{0,1\}$ such that

- If $\sigma$ is a leaf, $f(\sigma)=1$ if and only if $X$ is in the clopen set coded by $\ell(\sigma)$.

- If $\sigma$ is a union node, $f(\sigma)=1$ if and only if $f\left(\sigma^{\frown} n\right)=1$ for some $n \in \omega$.

- If $\sigma$ is an intersection node, $f(\sigma)=1$ if and only if $f\left(\sigma^{\urcorner} n\right)=1$ for all $n \in \omega$.

We say that $X$ is in the set coded by $T$, denoted $X \in|T|$, if there is an evaluation map $f$ for $X$ in $T$ such that $f(\lambda)=1$.

Note that $X \in|T|$ is a $\Sigma_{1}^{1}$ statement. Because evaluation maps are naturally constructed by arithmetic transfinite recursion, $\mathrm{ATR}_{0}$ proves that if $T$ is a Borel

\footnotetext{
${ }^{1}$ The original definition of a Borel code in reverse mathematics [Sim09] is a well-founded tree $T$ such that for exactly one $m \in \omega,\langle m\rangle \in T$.

Some conventions are then adopted: if $\langle m\rangle \in T$ is a leaf, then $T$ represents a clopen set coded by $m$ according to a standard computable look-up; if $\langle m\rangle$ is not a leaf, then $T$ represents a union or intersection according to the parity of $m$, and the sets to be thus combined are those coded by the subtrees $\left.T_{n}=\{\langle n\rangle\urcorner \sigma:\langle m, n\rangle \frown \sigma \in T\right\}$. Classically, one can translate easily between this definition and the definition of Borel code given above, but one direction of the translation requires $\mathrm{ACA}_{0}$ because one cannot effectively determine when a node is a leaf. All the principles considered in this paper will imply $\mathrm{ACA}_{0}$ over $\mathrm{RCA}$, so nothing will be muddled, but for the sake of fastidious readers, we will always call these labeled Borel codes to acknowledge the distinction.
} 
code and $X \in 2^{\omega}$, there is an evaluation map $f$ for $X$ in $T$. Furthermore, ACA $_{0}$ proves that if an evaluation map exists, then it is unique. For detailed proofs, see Sim09, Chapter V.3].

Because we are considering these definitions in the context of reverse mathematics, there will sometimes be an ill-founded $T$ which a model thinks is well-founded. In these cases, the statement $X \in|T|$ is meaningful inside the model, or in the context of a proof inside second order arithmetic, but is not meaningful outside a model. However, the criteria defining what it means to be an evaluation map are absolute, so we can and will construct evaluation maps on ill-founded but otherwise coherent labeled Borel codes. If $T$ is ill-founded, we will never use the notation $|T|$ outside of a model. But if $T$ is well-founded, then every $X$ has a unique evaluation map in $T$. In that case we give the notation " $|T|$ " the obvious meaning of

$\{X$ : the unique evaluation map $f$ for $X$ in $T$ satisfies $f(\lambda)=1\}$

when we refer to it outside the context of a model.

Operations on Borel sets are carried out easily. Observe that the operation which corresponds to complementation on a labeled Borel code is primitive recursive: just swap all the $\cup$ and $\cap$ labels, and replace every clopen leaf label with its complementary label.

Definition 2.7. If $(T, \ell)$ is a labeled Borel code, let $\left(T, \ell^{c}\right)$ denote the labeled Borel code whose tree is the same, and whose labeling $\ell^{c}$ is complementary to $\ell$ as described above.

Continuing the abuse of notation, if $T$ is used to refer to some $(T, \ell)$, then $T^{c}$ will be shorthand for $\left(T, \ell^{c}\right)$. Observe that $\mathrm{RCA}_{0}$ proves that if $T$ is a labeled Borel code, then $T^{c}$ is a labeled Borel code. Similarly, if $\left(T_{n}\right)_{n \in \omega}$ is a sequence of labeled Borel codes, in $\mathrm{RCA}_{0}$ we can construct a code for the intersection or union of these sets in the obvious effective way, and $\mathrm{RCA}_{0}$ will prove that the result is a labeled Borel code.

2.6. On the maxim that "Borel sets need $A T R_{0}$ ". Because making meaning out of a standard (labeled) Borel code requires evaluation maps to be around, ATR is typically taken as the base theory when evaluating theorems involving Borel sets. Even when $\mathrm{ATR}_{0}$ is not taken as the base theory, theorems involving Borel sets tend to imply ATR . The probable reason for this was observed in [DFSW17.

Theorem 2.8 (DFSW17). In $\mathrm{RCA}_{0}$, the statement "For every Borel code $T$, there exists $X$ such that $X \in|T|$ or $X \in\left|T^{c}\right|$ " implies ATR $\mathrm{R}_{0}$.

The strength comes from the fact that this statement is asserting the existence of an evaluation map for $X$ in $T$. If $f$ is an evaluation map for $X$ in $T$, then $1-f$ is an evaluation map for $X$ in $T^{c}$ 色

Restatement (of Theorem 2.8). The statement "For every Borel set, either it or its complement is nonempty" is equivalent to $\mathrm{ATR}_{0}$ over $\mathrm{RCA}_{0}$.

This can make the reverse mathematics of some standard theorems about Borel sets feel rather empty. Here is an example. Recall that a set $A \subseteq 2^{\omega}$ has the property of Baire if it differs from an open set by a meager set. That is, there

\footnotetext{
${ }^{2}$ The statement in DFSW17 is for original Borel codes, but the proof of the theorem remains valid for labeled Borel codes.
} 
are open sets $U$ and $\left\{D_{n}\right\}_{n \in \omega}$ such that each $D_{n}$ is dense, and for all $X \in \cap_{n} D_{n}$, $X \in U \Leftrightarrow X \in A$. A basic proposition is that every Borel set has the property of Baire, but what is the strength of that proposition in reverse mathematics? In DFSW17, the relevant notions were formalized as follows.

Definition 2.9. A Baire code is a collection of open sets $U, V,\left\{D_{n}\right\}_{n \in \omega}$ such that $U \cap V=\emptyset$ and the sets $U \cup V$ and $D_{n}$ are dense.

The statement PB below formalizes the proposition "Every Borel set has the property of Baire."

Definition 2.10. If $T$ is a Borel code and $U, V,\left\{D_{n}\right\}$ is a Baire code, we say that $U, V,\left\{D_{n}\right\}$ is a Baire approximation to $T$ if for all $X \in \cap_{n} D_{n}, X \in U \Rightarrow X \in|T|$ and $X \in V \Rightarrow X \in\left|T^{c}\right|$.

Definition 2.11. Let PB denote the statement "Every Borel code has a Baire approximation."

Proposition 2.12. DFSW17] In $\mathrm{RCA}_{0}, \mathrm{ATR}_{0}$ is equivalent to PB.

Proof. $(\Rightarrow)$ The standard proof uses arithmetic transfinite recursion.

$(\Leftarrow)$ If a set has the property of Baire, either it or its complement is nonempty.

The reverse direction of this proof is highly unsatisfactory. The purpose of this paper is to propose a variant on the definition of a Borel set which avoids this and similar unsatisfactory reversals to $\mathrm{ATR}_{0}$.

2.7. Some landmarks between $\mathrm{ATR}_{0}$ and $\mathrm{Jl}$. We will end up placing a variant of PB somewhere in a zoo which exists just below $A T R_{0}$. Much of what is known about this region concerns theories, such as $\Delta_{1}^{1}-\mathrm{CA}_{0}$, whose $\omega$-models are closed under join, hyperarithmetic reduction, and not much more.

Definition 2.13. A statement of hyperarithmetic analysis is any statement $S$ such that

(1) whenever $\mathcal{M}$ is an $\omega$-model which satisfies $S$, its second-order part $M$ is closed under hyperarithmetic reduction.

(2) For every $Y, H Y P(Y)$ is the second-order part of an $\omega$-model of $S$, where $H Y P(Y)=\left\{X: X \leq_{h} Y\right\}$.

$A$ theory of hyperarithmetic analysis is any theory which satisfies the same requirements as above.

The original definition of a theory of hyperarithmetic analysis, due to Steel Ste78, was a theory whose minimum $\omega$-model is $H Y P$. The relativized version above was first explicitly defined in [Mon06. At the time of Steel's definition, theories such as $\Delta_{1}^{1}-\mathrm{CA}_{0}$ were unrelativized (did not permit real parameters from the model). However, modern definitions of these theories allow parameters. Therefore, the relativized definition of theories of hyperarithmetic analysis is the right one for modern usage.

It would be tempting to hope that there would be some theory of hyperarithmetic analysis whose $\omega$-models are exactly the Turing ideals which are closed under hyperarithmetic reduction, in analogy to the theorems characterizing the $\omega$-models of $\mathrm{RCA}_{0}$ as the Turing ideals, the $\omega$-models of $\mathrm{WKL}_{0}$ as the Scott ideals, and the $\omega$-models of $\mathrm{ACA}_{0}$ as the Turing ideals closed under arithmetic reduction. However, no such theory can exist. 
Theorem 2.14. VW77] For every theory $T$, all of whose $\omega$-models are closed under hyperarithmetic reduction, there is a strictly weaker theory $T^{\prime}$, all of whose $\omega$-models are also closed under hyperarithmetic reduction, and which has more $\omega$ models than $T$.

Therefore, we are stuck with an infinitely descending zoo of statements/theories of hyperarithmetic analysis.

One theory of hyperarithmetic analysis is most relevant to us. Recall that a formula of $L_{\omega_{1}, \omega}$ is a formula constructed from the usual building blocks of firstorder logic, together with countably infinite conjunctions and disjunctions. In a language which contains no atomic formulas other than true and false, a formula of $L_{\omega_{1}, \omega}$ is just a well-founded tree whose interior nodes are labeled with either $U$ (infinite disjunction) or $\cap$ (infinite conjunction), and whose leaves are labeled with either true or false. An evaluation map for a formula of $L_{\omega_{1}, \omega}$ is defined the same as an evaluation map for an element $X$ in a Borel code $T$, except that the evaluation map must satisfy $f(\sigma)=1$ if $\ell(\sigma)=$ true and $f(\sigma)=0$ if $\ell(\sigma)=$ false. A formula of $L_{\omega_{1}, \omega}$ is completely determined if it has an evaluation map. Classically, every formula of $L_{\omega_{1}, \omega}$ is completely determined, but in weaker theories the witnessing function could fail to exist. A formula is called true if it has a witnessing function which maps the formula itself to true.

The following definition and result essentially appear in [Mon06, where $L_{\omega_{1}, \omega}-C A$ goes by the name CDG-CA, and is stated in terms of games. The name $\mathrm{L}_{\omega_{1}, \omega}-\mathrm{CA}$ and the definition given here were introduced in [Mon09.

Definition 2.15 (similar to Mon06]). The principle $\mathrm{L}_{\omega_{1}, \omega}-\mathrm{CA}$ is this statement: If $\left\{\phi_{i}: i \in \mathbb{N}\right\}$ is a sequence of completely determined $L_{\omega_{1}, \omega}$ formulas, then the set $X=\left\{i: \phi_{i}\right.$ is true $\}$ exists.

Theorem 2.16 (essentially [Mon06]). The principle $\mathrm{L}_{\omega_{1}, \omega}-\mathrm{CA}$ is a statement of hyperarithmetic analysis.

2.8. Genericity. The concept of genericity stems directly from category; a sufficiently generic member of a set which has the property of Baire has individual behavior that agrees with the behavior of a comeager set of reals. In this subsection we introduce the terminology around genericity and provide proofs of several folklore results which will be needed later.

A predicate $P(X)$ is called computable if there is a Turing functional $\Delta$ such that for all $X \in 2^{\omega}, \Delta(X)$ halts and outputs true or false according to the truth value of $P(X)$. A relativized formula of $L_{\omega_{1}, \omega}$ is a formula of $L_{\omega_{1}, \omega}$ for which the leaves bear computable predicates instead of simply true or false. Using the compactness of $2^{\omega}$ to translate between clopen sets and $\{X: \Delta(X)=$ true $\}$, it is immediate that the set of $X$ which satisfy a given relativized formulas of $L_{\omega_{1}, \omega}$ are exactly the members of the Borel set coded by essentially the same formula. If such a formula $\phi$ is computable, $a$-ranked, and has a union at the root, then $\left\{X \in 2^{\omega}: \phi(X)\right\}$ is a $\Sigma_{a}^{0}$ set and the formula is called a $\Sigma_{a}^{0}$ formula. Of course, the input to the formula could also be a natural number, in which case it defines a $\Sigma_{a}^{0}$ subset of $\omega$.

If $S \subseteq 2^{\omega}$ is a set of strings, a real $X$ is said to meet $S$ if for some $p \in S, p \prec X$, while $X$ is said to avoid $S$ if some $p \prec X$ has no extension in $S$. The set $S$ is dense if every $p \in 2^{<\omega}$ can be extended to meet it. A real $X$ is called a-generic if $X$ meets 
or avoids every $\Sigma_{a}^{0}$ set of strings. The following propositions, which taken together informally assert that set of strings which force a $\Sigma_{a}^{0}$ statement is $\Sigma_{a}^{0}$, are folklore.

Proposition 2.17. Uniformly in a code for a $\Sigma_{a}^{0}$ set $A$, there is a $\Sigma_{a}^{0}$ code for an open set $U$, as well as a uniform sequence of $\Sigma_{a}^{0}$ codes for dense open sets $D_{n}$ such that for all $X \in \cap_{k} D_{k}$, we have $X \in A$ if and only if $X \in U$.

Proof. This is a straightforward effectivization of the usual proof that every Borel set has the property of Baire.

The result is is immediate if $a$ is the 0 ordinal (in which case all sets described are clopen). Suppose that it holds for all $b<_{*} a$. We have $A=\cup_{n} A_{n}$ where each $A_{n}$ is $\Pi_{b_{n}}^{0}$ for some $b_{n}<_{*} a$. Apply the induction hypothesis to the complements $A_{n}^{c}$ to get a sequence of open sets $U_{n}$ and a double sequence of dense open sets $D_{n, k}$, where each $U_{n}$ and $D_{n, k}$ have a $\Sigma_{b_{n}}^{0}$ code, and any $X \in \cap_{k} D_{n, k}$ is in $A_{n}^{c}$ if and only if it is in $U_{n}$. Define $V_{n}$ to be the interior of $U_{n}^{c}$. Then each $V_{n}$ is uniformly $\Sigma_{b_{n}+1}^{0}$ and thus $\Sigma_{a}^{0}$. We can let $U=\cup_{n} V_{n}$ and let the sequence of dense open sets include all sets $D_{n, k}$, as well as sets of the form $U_{n} \cup V_{n}$. Suppose that $X$ meets all these dense sets. The $X \in A$ exactly if $X \in A_{n}$ for some $n$, which happens exactly if $X \notin U_{n}$ for some $n$. Since $X \in U_{n} \cup V_{n}$, this is true exactly when $X \in V_{n}$ for some $n$, equivalently when $X \in U$.

Proposition 2.18. If $\phi(X, q)$ is a $\Sigma_{a}^{0}$ formula, there is a $\Sigma_{a}^{0}$ formula $R(q)$ such that for all a-generic reals $X$,

$$
\{q: \phi(X, q)\}=\{q: \exists n R(X \nmid n, q)\}
$$

Proof. By the previous proposition, uniformly in $q$ there is a code for a $\Sigma_{a}^{0}$ set $U_{q} \subseteq 2^{<\omega}$ and a sequence of $\Sigma_{a}^{0}$ sets $D_{k} \subseteq 2^{<\omega}$ such that each $D_{k}$ is dense and for any $X$ that meets each $D_{k}, X$ meets $U_{q}$ if and only if $\phi(X, q)$. Thus $R(r, q)$ can be taken to be $\exists p\left(p \in U_{q}\right.$ and $\left.p \prec r\right)$.

Now we review some notions from higher genericity. We assume a general familiarity with hyperarithmetic theory, and refer the reader to Sac90 for definitions and details. For $G \in 2^{\omega}$, it is well-known that an element $X$ of $2^{\omega}$ is $\Delta_{1}^{1}(G)$ if and only if it is $\operatorname{HYP}(G)$, if and only if there is some $b \in \mathcal{O}^{G}$ such that $X \leq_{T} H_{b}^{G}$.

Recall that if $\Gamma$ is a pointclass, $X \in 2^{\omega}$ is called $\Gamma$-generic if $X$ meets or avoids every open set $U$ with a code in $\Gamma$. (We have already seen this in the case $\Gamma=\Sigma_{a}^{0}$.) We are interested in $\Delta_{1}^{1}$-generics $G$ with the additional property that $\omega_{1}^{c k}=\omega_{1}^{G}$. By [GM17, these are precisely the $\Sigma_{1}^{1}$-generics. However, for our purposes the formal definition of $\Sigma_{1}^{1}$-genericity seems less useful than the " $\Delta_{1}^{1}$-generic and $\omega_{1}^{c k}$ preserving", and indeed we never use the equivalence with $\Sigma_{1}^{1}$-genericity in any way other than as an (accurate) notational shorthand.

The following three propositions must be folklore, but we give their proofs here. Recall that $A$ and $B$ are relatively $\Gamma$-generic if $A$ is $\Gamma(B)$-generic and $B$ is $\Gamma(A)$ generic.

Proposition 2.19. For $G_{0}, G_{1} \in 2^{\omega}$, we have $G_{0} \oplus G_{1}$ is $\Sigma_{1}^{1}$-generic if and only if $G_{0}$ and $G_{1}$ are relatively $\Sigma_{1}^{1}$-generic.

Proof. Consider the argument in [DH10, Thm. 8.20.1] (originally due to [Yu6]), where it is shown that $A \oplus B$ is $n$-generic if and only if $A$ and $B$ are relatively $n$-generic. Observe that at no point do they make use of the fact that $n$ is finite, and the same argument goes through if $n$ is replaced with any $a \in \mathcal{O}$. (Proposition 
2.18 is used in the $a \in \mathcal{O}$ case.) Therefore the same argument shows that $A \oplus B$ is $a$-generic if and only if $A$ and $B$ are relatively $a$-generic. Observe that $A$ is $\Delta_{1}^{1}$-generic if and only if $A$ is $a$-generic for all $a \in \mathcal{O}$.

Now suppose that $G_{0} \oplus G_{1}$ is $\Sigma_{1}^{1}$-generic. We will show that $G_{0}$ is $\Sigma_{1}^{1}\left(G_{1}\right)$-generic. We have $\omega_{1}^{G_{0} \oplus G_{1}}=\omega_{1}^{c k}=\omega_{1}^{G_{1}}$, so it suffices to show that $G_{0}$ is $\Delta_{1}^{1}\left(G_{1}\right)$-generic, or equivalently, that $G_{0}$ is $a$-generic relative to $G_{1}$ for all $a \in \mathcal{O}$ (here we use the fact that $\left.\omega_{1}^{G_{1}}=\omega_{1}^{c k}\right)$. This follows from the previous paragraph because $G_{0} \oplus G_{1}$ is $a$-generic.

On the other hand, if $G_{0}$ and $G_{1}$ are relatively $\Sigma_{1}^{1}$-generic, then in particular each is $\Sigma_{1}^{1}$-generic, so $\omega_{1}^{G_{0}}=\omega_{1}^{G_{1}}=\omega_{1}^{c k}$, and by relative $\Sigma_{1}^{1}$-genericity, we also have $\omega_{1}^{G_{0} \oplus G_{1}}=\omega_{1}^{c k} 3$ Therefore it suffices to show that $G_{0} \oplus G_{1}$ is $\Delta_{1}^{1}$-generic, or equivalently, that it is $a$-generic for all $a \in \mathcal{O}$. This follows because $G_{0}$ and $G_{1}$ are relatively $a$-generic for all $a \in \mathcal{O}$.

The following two propositions will be used later in a relativized form. To reduce clutter, we do not write the relativized form, but the reader can verify that all the arguments relativize.

Proposition 2.20. If $G_{0} \oplus G_{1}$ is $\Sigma_{1}^{1}$-generic, then $\Delta_{1}^{1}\left(G_{0}\right) \cap \Delta_{1}^{1}\left(G_{1}\right)=\Delta_{1}^{1}$.

Proof. If $X \in \Delta_{1}^{1}\left(G_{0}\right) \cap \Delta_{1}^{1}\left(G_{1}\right)$, then since $\omega_{1}^{G_{0}}=\omega_{1}^{G_{1}}=\omega_{1}^{c k}$, there are $a \in \mathcal{O}$ and indices $e$ and $f$ such that $X=\Phi_{e}\left(H_{a}^{G_{0}}\right)=\Phi_{f}\left(H_{a}^{G_{1}}\right)$. Consider the set

$$
W=\left\{Y \oplus Z: \Phi_{e}\left(H_{a}^{Y}\right)=\Phi_{f}\left(H_{a}^{Z}\right)\right\} .
$$

This set is $\Delta_{1}^{1}$, so it has the property of Baire, and in particular there is a $\Delta_{1}^{1}$ open set $V$ such that every sufficiently generic $Y \oplus Z$ is an element of $V$ if and only if it is an element of $W$. Here the amount of genericity needed is not full $\Delta_{1}^{1}$-genericity, but rather $c$-genericity, where $c=a+O(1)$. To see that $c$-genericity suffices, first use Proposition 2.1 to write the defining property of $W$ as a $c$-ranked relativized formula of $L_{\omega_{1}, \omega}$, then apply Proposition 2.18 .

Since $G_{0} \oplus G_{1}$ is $\Delta_{1}^{1}$-generic and in $W$, it is in $V$. Let $p, q \in 2^{<\omega}$ be such that $p \prec G_{0}, q \prec G_{1}$ and $p \oplus q \in V$. Now let $Y$ be any $c$-generic, hyperarithmetic real with $p \prec Y$. Then since $G_{1}$ is $\Delta_{1}^{1}$-generic, it is $\Delta_{1}^{1}$-generic relative to $Y$, so in particular it is $c$-generic relative to $Y$. Using the ordinal version of [DH10, Thm. 8.20.1] a second time, we conclude that $Y \oplus G_{1}$ is $c$-generic, and meets $V$. Therefore, $Y \oplus G_{1} \in W$, and we obtain a $\Delta_{1}^{1}$ formula for $X$, that is, $X=\Phi_{e}\left(H_{a}^{Y}\right)$.

Proposition 2.21. Let $G_{0}$ be $\Sigma_{1}^{1}$-generic and $P$ a hyperarithmetic predicate. If there is a $Y \in \Delta_{1}^{1}\left(G_{0}\right)$ such that $P(Y)$ holds, then for all $\Delta_{1}^{1}$-generic $G_{1}$, there is a $Y \in \Delta_{1}^{1}\left(G_{1}\right)$ such that $P(Y)$ holds.

Proof. Since $\omega_{1}^{c k}=\omega_{1}^{G_{0}}$, there is some $a \in \mathcal{O}$ and an index $e$ such that $Y=$ $\Phi_{e}\left(H_{a}^{G_{0}}\right)$. Then $R(X):=\exists e P\left(\Phi_{e}\left(H_{a}^{X}\right)\right)$ is a hyperarithmetic predicate that holds of $G_{0}$ and holds of $p^{\frown} G_{0}$ for any $p \in 2^{<\omega}$. Therefore, for any $\Delta_{1}^{1}$-generic $G_{1}, R\left(G_{1}\right)$ holds.

\footnotetext{
${ }^{3}$ Although [GM17 does relativize, the conclusions here can be established without using that relativization. It suffices to show that at least one of $G_{0}, G_{1}$ is $\omega_{1}^{c k}$ preserving. Suppose that $G_{0}$ computes a linear order of order type $\omega_{1}^{c k}$. Then $\{X$ : $X$ computes a linear order of order type $\left.\omega_{1}^{c k}\right\}$ is $\Delta_{1}^{1}\left(G_{0}\right)$ and meager, so $G_{1}$ does not belong to it. Thus $\omega_{1}^{G_{1}}=\omega_{1}^{c k}$.
} 
Finally, we remark that for any $Z$, the set of $\Delta_{1}^{1}(Z)$-generics is $\Sigma_{1}^{1}(Z)$. This is because

$$
X \text { is } \Delta_{1}^{1}(Z) \text {-generic } \Longleftrightarrow \forall Y \in \Delta_{1}^{1}(Z)[X \text { is } 1 \text {-generic relative to } Y] \text {. }
$$

\section{Completely Determined Borel Codes}

We propose the following variation on the definition of a Borel code. We shall see that when this variant is used, the unsatisfactory shortcut in Proposition 2.12 vanishes, and indeed the reversal no longer holds.

Definition 3.1. A labeled Borel code $T$ is called completely determined if every $X \in 2^{\omega}$ has an evaluation map in T. A completely determined Borel code is a labeled Borel code that is completely determined.

When we formalize statements in reverse mathematics, in order to not conflict with existing convention, we will say completely determined Borel set to indicate when the formalized version of the statement should call for a completely determined Borel code.

The following facts are immediate.

\section{Proposition 3.2. In $\mathrm{RCA}_{0}$,}

(1) If $T$ is a completely determined Borel code, then $T^{c}$ is also a completely determined Borel code.

(2) For every completely determined Borel set $A$ and $X \in 2^{\omega}$, either $X \in A$ or $X \notin A$.

With only a slight amount of effort, we also have the following.

Proposition 3.3. In $\mathrm{RCA}_{0}$, if $A$ is a completely determined Borel set and $h: 2^{\omega} \rightarrow$ $2^{\omega}$ is continuous, then $h^{-1}(A)$ is a completely determined Borel set.

Proof. Let $T$ be a completely determined Borel code and $h: 2^{\omega} \rightarrow 2^{\omega}$ a continuous function. Then $h$ is encoded by a sequence of pairs $\left(p_{1}, q_{1}\right),\left(p_{2}, q_{2}\right) \ldots$ from $2^{<\omega} \times 2^{<\omega}$, which are compatible in the sense that $p \preceq p^{\prime} \Longrightarrow q \preceq q^{\prime}$ whenever $(p, q),\left(p^{\prime}, q^{\prime}\right)$ are in $h$. If $(p, q)$ is in $h$, it means that $p \prec X$ implies that $q \prec h(X)$. For $h$ to be well-defined, we must have that for each $X$, there are arbitrarily long $q$ for which $q \prec h(X)$. Define $S$ by starting with $S=T$ and modifying each leaf $\sigma \in T$ as follows:

(1) In $S, \sigma$ is a union.

(2) For each $n, \sigma^{\urcorner} n \in S$ and is a leaf.

(3) If $U$ is the clopen set attached to $\sigma$ in $T$, let $\sigma^{\wedge} n$ be labeled with a code for the clopen subset of $h^{-1}(U)$ defined by

$$
\cup\left\{\left[p_{i}\right]:\left(p_{i}, q_{i}\right) \in h, i<n,\left[q_{i}\right] \subseteq U\right\}
$$

We claim that $S$ is completely determined and $X \in|S|$ if and only if $h(X) \in|T|$. Let $f$ be an evaluation map for $h(X)$ in $T$. We claim that $f$ can be extended to an evaluation map for $X$ in $S$ by adding $f\left(\sigma^{\wedge} n\right)=1$ if and only if $X$ is in the clopen set attached to $\sigma^{\wedge} n$ in $S$. One only needs to check that the logic of the evaluation map is correct at each $\sigma$ which was a leaf in $T$.

The fact that Borel sets are closed under countable union, which was trivial using the standard definition of a Borel set, has quite some power for completely determined Borel sets. 
Proposition 3.4. In $\mathrm{RCA}_{0}$, the statement "A countable union of completely determined Borel sets is a completely determined Borel set" is equivalent to $\mathrm{L}_{\omega_{1}, \omega}$-CA.

Proof. If $\left\{T^{k}: k \in \mathbb{N}\right\}$ are completely determined Borel codes, and $T=\{\lambda\} \cup$ $\left.\{\langle k\rangle\urcorner \sigma: \sigma \in T^{k}\right\}$, we claim that, assuming $\mathrm{L}_{\omega_{1}, \omega}-\mathrm{CA}, T$ is completely determined. Fixing $X$, let $\phi_{k, \sigma}$ be the formula obtained by replacing each clopen set at each leaf of $T_{\sigma}^{k}$ by true or false according to whether $X$ is in each clopen set. Any evaluation map for $X$ in $T^{k}$ can be restricted to an evaluation map for $X$ in $T_{\sigma}^{k}$, which is an evaluation map for $\phi_{k, \sigma}$, so all these formulas are completely determined. One obtains an evaluation map for $X$ in $T$ by letting $f(\sigma)=1$ if and only if $\phi_{k, \sigma}$ is true, and then non-uniformly filling in $f(\lambda)$ to its unique correct value.

Conversely, if $\left\{\phi_{k}: k \in \mathbb{N}\right\}$ are completely determined, these formulas can be modified at the leaves to become completely determined Borel codes $T^{k}$ for $\emptyset$ or $2^{\omega}$ according to whether they are true or false. Defining $T$ as above, any evaluation map $f$ for $T$ satisfies $f(\langle k\rangle)=1$ if and only if $\phi_{k}$ is true.

Now we consider the completely determined variant of PB.

Definition 3.5. Let CD-PB be the statement "Every completely determined Borel set has the property of Baire."

Our main question is: what is the reverse mathematics strength of CD-PB?

Proposition 3.6. In $\mathrm{RCA}_{0}$, CD-PB implies $\mathrm{L}_{\omega_{1}, \omega}-\mathrm{CA}$.

Proof. Any sequence $\left\{\phi_{k}: k \in \mathbb{N}\right\}$ of completely determined formulas of $L_{\omega_{1}, \omega}$ can be modified at the leaves to produce a sequence of completely determined Borel codes which code either $\left[0^{k} 1\right]$ or $\emptyset$ depending on whether $\phi_{k}$ is true or false. The union of these remains completely determined because each $X$ passes through at most one of these sets. Any Baire approximation to $\cup_{k: \phi_{k}}$ is true $\left[0^{k} 1\right]$ computes $\left\{k: \phi_{k}\right.$ is true $\}$.

This places CD-PB somewhere in the general area of $A T R_{0}$ and the theories of hyperarithmetic analysis. If CD-PB were equivalent to $L_{\omega_{1}, \omega}-C A$, our variant would be subject to the same kinds of critique that we made of the original definition (all the strength of the theorem coming essentially from Proposition 3.4). However, it turns out CD-PB is equivalent to none of the principles mentioned so far.

When considering how to show that CD-PB is strictly weaker than $A_{T R}$, it is informative to consider the usual proof that every Borel set has the property of Baire. This proof uses arithmetic transfinite recursion on the Borel code of the given set. It constructs not only a Baire code for the given set, but also Baire codes for all Borel sets used to build up the given one. Below, we give the name Baire decomposition to this extended object that $\mathrm{ATR}_{0}$ would have created. Superficially, CD-PB would seem weaker than the statement "every completely determined Borel set has a Baire decomposition", and one might wonder whether the additional information in the Baire decomposition carries any extra strength. The purpose of the rest of this section is to show that it does not (Proposition 3.8), and to mention exactly how a Baire approximation is constructively obtained from a Baire decomposition (Proposition 3.9). The point is that any model separating CD-PB from $\mathrm{ATR}_{0}$ will need another method of producing an entire Baire decomposition, not just the Baire approximation. 
Definition 3.7. Let $T$ be a completely determined Borel code. A Baire decompositon for $T$ is a collection of open sets $U_{\sigma}$ and $V_{\sigma}$ for $\sigma \in T$ such that for each $\sigma \in T$ and each $p \in 2^{<\omega}$,

(1) $U_{\sigma} \cup V_{\sigma}$ is dense and $U_{\sigma} \cap V_{\sigma}=\emptyset$,

(2) if $\sigma$ is a leaf, then $U_{\sigma}$ is dense in the clopen set $C$ coded by $\ell(\sigma)$ and $V_{\sigma}$ is dense in $C^{c}$,

(3) if $\sigma$ is a union node, then $U_{\sigma}$ is dense in $\bigcup_{n} U_{\sigma>n}$ and $\bigcup_{n} U_{\sigma \frown n}$ is dense in $U_{\sigma}$,

(4) if $\sigma$ is an intersection node, then $V_{\sigma}$ is dense in $\bigcup_{n} V_{\sigma \sim n}$ and $\bigcup_{n} V_{\sigma \frown n}$ is dense in $V_{\sigma}$.

Proposition $3.8\left(\mathrm{ACA}_{0}\right)$. CD-PB implies that every completely determined Borel set has a Baire decomposition.

Proof. Let $T$ be a completely determined Borel code. Informally, we partition the space into countably many disjoint clopen pieces (plus one limit point) and put an isomorphic copy of the set coded by $T_{\sigma}$ in the $\sigma$ th piece. Then we show that a Baire approximation to this disintegrated set can be translated back to a Baire decomposition for the original set coded by $T$.

More formally, for any $p \in 2^{<\omega}$, let $T[p]$ denote the labeled Borel code for $\left\{p^{\wedge} X: X \in|T|\right\}$. This is an effective operation on codes. Recall that each leaf codes a clopen set by a finite list $F \subseteq 2^{<\omega}$. By replacing each such $F$ with $\left\{p^{\frown} q: q \in F\right\}$, we achieve the desired effect.

For any $\sigma \in \omega^{<\omega}$, let $\lceil\sigma\rceil$ be a natural number which codes $\sigma$ in a canonical way. Define $S$ to be the labeled Borel code

$$
S=\{\lambda\} \cup\left\{\lceil\sigma\rceil^{\curvearrowright} \tau: \tau \in T_{\sigma}\left[0^{\lceil\sigma\rceil} 1\right], \sigma \in T\right\}
$$

where $\lambda$ is a $\cup$ and all other labels are inherited from the $T_{\sigma}\left[0^{\lceil\sigma\rceil} 1\right]$. Then $S$ is completely determined: for any $X$, if $X=0^{\omega}$, then the identically zero map is an evaluation map for $X$; if $X=0^{n} 1^{\wedge} Y$, then if $f$ is an evaluation map for $Y$ in $T$ and $n=\lceil\sigma\rceil$, an evaluation map $g$ for $X$ in $S$ can be defined by letting $g\left(\lceil\sigma\rceil^{\wedge} \tau\right)=f\left(\sigma^{\frown} \tau\right)$ on

$$
\left\{\lceil\sigma\rceil^{\wedge} \tau: \tau \in T_{\sigma}\left[0^{\lceil\sigma\rceil} 1\right]\right\}
$$

$g(\lambda)=f(\sigma)$, and $g$ identically zero elsewhere. Therefore, for all $Y$ and $\sigma$,

$$
0^{\lceil\sigma\rceil} 1^{\frown} Y \in|S| \Longleftrightarrow Y \in\left|T_{\sigma}\right| .
$$

Now suppose that $\left(U, V,\left\{D_{k}\right\}_{k \in \omega}\right)$ is a Baire approximation for $S$. Then define $U_{\sigma}=\left\{q: 0^{\lceil\sigma\rceil} 1^{\frown} q \in U\right\}$ and $V_{\sigma}=\left\{q: 0^{\lceil\sigma\rceil} 1^{\frown} q \in V\right\}$. We claim that $\left(U_{\sigma}, V_{\sigma}\right)_{\sigma \in T}$ is a Baire decomposition for $T$. Property (1) of a Baire decomposition is clear. For property (2), this follows because if $[q]$ is contained in the clopen set $\left|T_{\sigma}\right|$, suppose for contradiction that there is $r$ extending $q$ with $\left[0^{\lceil\sigma\rceil} 1^{\frown} r\right] \subseteq V$. Then for all $X \in[r]$, we have $X \in\left|T_{\sigma}\right|$ and thus $0^{\lceil\sigma\rceil} 1^{\frown} X \in U$, a contradiction. Therefore $V_{\sigma} \cap[q]=\emptyset$, so $U_{\sigma}$ is dense in $[q]$. A similar argument applies to establish that $V_{\sigma}$ is dense in $[q]$ if $[q]$ is contained $\left|T_{\sigma}^{c}\right|$. For property (3), letting $\sigma$ be a union node and $p \in 2^{<\omega}$, we will show that $U_{\sigma}$ is dense in $[p]$ if and only if $\cup_{n} U_{\sigma \wedge n}$ is dense in $[p]$. Suppose that $\cup_{n} U_{\sigma \frown n}$ is not dense in $[p]$. Let $q$ extend $p$ such that for all $n, U_{\sigma \frown n} \cap[q]=\emptyset$. Then define $Y$ so that $q \prec Y$ and the following collection of comeager events occur:

(i) For all $n, Y \in V_{\sigma^{\curvearrowright} n}$ 
(ii) For all $n, 0^{\left\lceil\sigma^{\curvearrowright} n\right\rceil} 1^{\wedge} Y \in \cap_{k} D_{k}$

(iii) $Y \in U_{\sigma} \cup V_{\sigma}$

(iv) $0^{\lceil\sigma\rceil} 1^{\frown} Y \in \cap_{k} D_{k}$

The first comeager event guarantees that $Y \in V_{\sigma^{\wedge} n}$ for all $n$. Together with second comeager event this implies that $0^{\left\lceil\sigma^{\curvearrowright} n\right\rceil} 1^{\wedge} Y \notin|S|$, and therefore $Y \notin\left|T_{\sigma^{\wedge} n}\right|$. Therefore, $Y \notin\left|T_{\sigma}\right|$. In the third dense event, if we had $Y \in U_{\sigma}$, the fourth comeager event would imply that $Y \in\left|T_{\sigma}\right|$; therefore it must be that $Y \in V_{\sigma}$, and so $U_{\sigma}$ is not dense in $[p]$. On the other hand, if $U_{\sigma}$ is not dense in $[p]$, then assuming $\cup_{n} U_{\sigma\urcorner n}$ is dense in $[p]$ leads to a contradiction, for we may similarly define $Y$ to meet $V_{\sigma} \cap[p]$ and $\cup_{n} U_{\sigma \sim n}$, while also satisfying (iii) and (iv).

The proof of (4) is similar to the proof of (3).

Turning a Baire decomposition into a Baire approximation involves extracting the comeager set on which the approximation should hold. The following proposition gives a canonical sequence of dense open sets which suffices for this.

Proposition $3.9\left(\mathrm{ACA}_{0}\right)$. Let $T$ be a completely determined Borel code and $\left(U_{\sigma}, V_{\sigma}\right)_{\sigma \in T}$ be a Baire decomposition for $T$. Let $\left\{D_{n}\right\}_{n \in \omega}$ consist of the following dense open sets:

(1) $U_{\sigma} \cup V_{\sigma}$ for $\sigma \in T$,

(2) $V_{\sigma} \cup \bigcup_{n} U_{\sigma>n}$ for $\sigma \in T$ a union node, and

(3) $U_{\sigma} \cup \bigcup_{n} V_{\sigma \wedge n}$ for $\sigma \in T$ an intersection node.

Then, $\left(U_{\lambda}, V_{\lambda},\left\{D_{n}\right\}_{n \in \omega}\right)$ is a Baire approximation for $T$.

Proof. The properties of a Baire decomposition suffice to ensure that $\left(U_{\lambda}, V_{\lambda},\left\{D_{n}\right\}_{n \in \omega}\right)$ is a Baire code. We must show that if $X \in \cap_{n} D_{n}$, then $X \in U_{\lambda} \Longrightarrow X \in|T|$ and $X \in V_{\lambda} \Longrightarrow X \in|T|^{c}$. Fix $X \in \cap_{n} D_{n}$. We prove by arithmetic transfinite induction that for all $\sigma \in T$, if $X \in U_{\sigma}$ then $X \in\left|T_{\sigma}\right|$ and if $X \in V_{\sigma}$ then $X \in\left|T_{\sigma}^{c}\right|$. This holds when $\sigma$ is a leaf.

If $\sigma$ is a union node, suppose $X \in U_{\sigma}$. Then $X \notin V_{\sigma}$, but $X \in V_{\sigma} \cup \bigcup_{n} U_{\sigma^{\wedge} n}$, so $X \in U_{\sigma^{\wedge} n}$ for some $n$. Then the induction hypothesis gives us $X \in\left|T_{\sigma^{\wedge} n}\right|$, so $X \in\left|T_{\sigma}\right|$ since $\sigma$ is a union node.

On the other hand, if $X \in V_{\sigma}$, let $p \prec X$ be such that $p \in V_{\sigma}$. Then $U_{\sigma} \cap[p]=\emptyset$, so $\cup_{n} U_{\sigma^{\frown} n} \cap[p]=\emptyset$. Recall that $U_{\sigma^{\wedge} n} \cup V_{\sigma^{\wedge} n}$ is dense by definition. So for each $n, V_{\sigma \frown n}$ is dense in $[p]$. Therefore, $X$ meets each $V_{\sigma \frown n}$, so by induction $X \in\left|T_{\sigma \frown n}^{c}\right|$ holds for all $n$. Therefore, $X \in\left|T_{\sigma}^{c}\right|$.

The case where $\sigma$ is an intersection node is similar.

\section{CD-PB DOES NOT IMPLY ATR}

Our non-ATR $\mathrm{R}_{0}$ method of producing a Baire decomposition involves polling sufficiently generic $X$ to see whether they are in or out of a given set. For our purposes, sufficiently generic means $\Sigma_{1}^{1}$-generic.

Let $G=\bigoplus_{i} G_{i}$ be a $\Sigma_{1}^{1}$ generic. Let $\mathcal{M}=\bigcup_{n} \Delta_{1}^{1}\left(\bigoplus_{i<n} G_{i}\right)$. This is the model

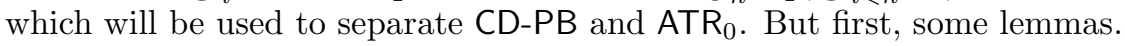

Lemma 4.1. $\mathcal{M} \models \mathrm{L}_{\omega_{1}, \omega}$-CA. Furthermore, whenever $F \subseteq \omega$ is finite and the completely determined sequence of formulas $\left\{\phi_{k}: k \in \mathbb{N}\right\}$ is in $\Delta_{1}^{1}\left(\bigoplus_{i \in F} G_{i}\right)$, we also have

$$
\left\{k: \phi_{k} \text { is true in } M\right\} \in \Delta_{1}^{1}\left(\bigoplus_{i \in F} G_{i}\right) \text {. }
$$


Proof. We begin with three facts. First, applying Proposition 2.19 to the decomposition $G=\bigoplus_{i \in F} G_{i} \oplus \bigoplus_{i \notin F} G_{i}$, we conclude that $\bigoplus_{i \notin F} G_{i}$ is $\Sigma_{1}^{1}\left(\bigoplus_{i \in F} G_{i}\right)$-generic.

Second, fix $j \notin F$. Applying Proposition 2.19 to $G=G_{j} \oplus \bigoplus_{i \neq j} G_{i}$, we have that $G_{j}$ is $\Sigma_{1}^{1}\left(\bigoplus_{i \neq j} G_{i}\right)$-generic and hence $G_{j}$ is $\Sigma_{1}^{1}\left(\bigoplus_{i \in F} G_{i}\right)$-generic.

Third, fix $j_{0}, j_{1} \notin F$ with $j_{0} \neq j_{1}$. By the same argument, we have that $G_{j_{0}}$ is $\Sigma_{1}^{1}\left(G_{j_{1}} \oplus \bigoplus_{i \in F} G_{i}\right)$-generic and that $G_{j_{1}}$ is $\Sigma_{1}^{1}\left(G_{j_{0}} \oplus \bigoplus_{i \in F} G_{i}\right)$-generic. By Proposition 2.20 relativized to $\bigoplus_{i \in F} G_{i}$, it follows that $\Delta_{1}^{1}\left(G_{j_{0}} \oplus \bigoplus_{i \in F} G_{i}\right) \cap \Delta_{1}^{1}\left(G_{j_{1}} \oplus\right.$ $\left.\bigoplus_{i \in F} G_{i}\right)=\Delta_{1}^{1}\left(\bigoplus_{i \in F} G_{i}\right)$.

We now apply Proposition 2.21 relativized to $\bigoplus_{i \in F} G_{i}$. Fix $j \notin F$ and $k \in \omega$. Since $\bigoplus_{i \notin F} G_{i}$ is $\Sigma_{1}^{1}\left(\bigoplus_{i \in F} G_{i}\right)$-generic, $G_{j}$ is $\Sigma_{1}^{1}\left(\bigoplus_{i \in F} G_{i}\right)$-generic and there is a $\Delta_{1}^{1}(G)$ evaluation map for $\phi_{k}$, it follows that $\phi_{k}$ is completely determined in $\Delta_{1}^{1}\left(G_{j} \oplus \bigoplus_{i \in F} G_{i}\right)$. Because this holds for any $j \notin F, \phi_{k}$ is completely determined in $\Delta_{1}^{1}\left(\bigoplus_{i \in F} G_{i}\right)$ by the third fact above. Since $\mathrm{L}_{\omega_{1}, \omega}$ CA is a theory of hyperarithmetic analysis, the conclusion follows.

Proposition 4.2. $\mathcal{M} \not \models \mathrm{ATR}_{0}$.

Proof. Let $a^{*} \in \mathcal{O}^{*}$. Then $\mathcal{M}$ believes that $a^{*}$ is an ordinal. For if there were a $\Delta_{1}^{1}(G)$-computable descending sequence in $a^{*}$, then for some $b \in \mathcal{O}$ (here we use the fact that $\omega_{1}^{c k}=\omega_{1}^{G}$ ) the statement $R(X)$ : " $H_{b}^{X}$ computes a descending sequence in $a^{*}$ " is a hyperarithmetic predicate which holds of $G$. As $R$ holds of $p^{\frown} G$ for any $p \in 2^{\omega}$, the set of $X$ for which $R$ holds is comeager (since each $p^{\frown} G$ is $\Sigma_{1}^{1}$-generic, there can be no $p$ which forces $\neg R(X)$, therefore the set of $p$ which force $R(X)$ is dense). Furthermore, $R(X)$ is $\Sigma_{b+O(1)}^{0}$, so $R(X)$ holds for any $X$ which is $b+O(1)$-generic. There is a hyperarithmetic such $X$. But then $H_{b}^{X}$ is also hyperarithmetic, contradicting that $a^{*}$ has no hyperarithmetic descending sequence. So $a^{*}$ is well-founded, according to $\mathcal{M}$.

For contradiction, suppose there were a jump hierarchy on $a^{*}$ in $\Delta_{1}^{1}(G)$. Then for some $b \in \mathcal{O}, R(X):=$ " $H_{b}^{X}$ computes a jump hierarchy on $a^{*}$ " is again a $\Sigma_{b+O(1)}^{0}$ predicate, where $R$ holds of $G$. (Recall that being a jump hierarchy on $a^{*}$ is just a $\Pi_{2}^{0}$ property). Arguing as above, hyperarithmetically in any $b+O(1)$-generic $X$, we would have a jump hierarchy on $a^{*}$, which is impossible since $a^{*}$ has no hyperarithmetic jump hierarchy.

Below, the way that $\mathcal{M}$ can produce a Baire decomposition without resorting to arithmetic transfinite recursion is by polling a sufficiently generic element $G_{i}$ about whether $p^{\complement} G_{i} \in|T|$ while varying $p \in 2^{<\omega}$ to get a complete picture of the comeager behavior of $T$.

Theorem 4.3. There is an $\omega$-model of CD-PB that does not satisfy $\mathrm{ATR}_{0}$.

Proof. Let $\mathcal{M}$ be as above. Let $T \in M$ be a labeled Borel code which is completely determined in $M$. We consider the case where $T \in \Delta_{1}^{1}$; the case where $T \in$ $\Delta_{1}^{1}\left(\bigoplus_{i<n} G_{i}\right)$ follows by relativization. Since $T$ is completely determined, for each $G_{i}$ and each $p \in 2^{<\omega}$, the statements $p^{\frown} G_{i} \in\left|T_{\sigma}\right|$ can be understood as a completely determined formulas of $L_{\omega_{1}, \omega}$ (by replacing the leaves of $T_{\sigma}$ with 0 or 1 according to whether $p^{\frown} G_{i}$ is in those sets). These formulas are uniformly $\Delta_{1}^{1}\left(G_{i}\right)$. Therefore, by Lemma 4.1, we have

$$
\left\{(\sigma, p): p^{\frown} G_{i} \in\left|T_{\sigma}\right|\right\} \in \Delta_{1}^{1}\left(G_{i}\right)
$$


Therefore, for each $i, \Delta_{1}^{1}\left(G_{i}\right)$ contains the sequence $\left(U_{\sigma}^{i}, V_{\sigma}^{i}\right)_{\sigma \in T}$ defined by

$$
U_{\sigma}^{i}=\left\{p: \forall q \succeq p, q^{\frown} G_{i} \in\left|T_{\sigma}\right|\right\}, \quad V_{\sigma}^{i}=\left\{p: \forall q \succeq p, q \notin U_{\sigma}^{i}\right\} .
$$

We claim that for each $i \neq j$ and for each $\sigma \in T$, the collections

$$
\left(U_{\sigma \frown \tau}^{i}, V_{\sigma \frown \tau}^{i}\right)_{\tau \in T_{\sigma}}, \quad\left(U_{\sigma \wedge \tau}^{j}, V_{\sigma \wedge \tau}^{j}\right)_{\tau \in T_{\sigma}}
$$

are Baire decompositions for $T_{\sigma}$, and are equal. The proof (for fixed $i, j$ ) is carried out inside of $\mathcal{M}$ by arithmetic transfinite induction on the rank of $\sigma$ in $T$. Specifically, we claim that

(1) If $\sigma$ is a leaf, then $U_{\sigma}^{i}=$ the clopen set coded by $\sigma$ and $V_{\sigma}=\left(U_{\sigma}^{i}\right)^{c}$.

(2) If $\sigma$ is a union node, then for all $p \in 2^{<\omega}, p \in U_{\sigma}^{i}$ if and only if $\bigcup_{n} U_{\sigma \wedge n}^{i}$ is dense in $[p]$.

(3) If $\sigma$ is an intersection node, then for all $p \in 2^{<\omega}, p \in V_{\sigma}^{i}$ if and only if $\bigcup_{n} V_{\sigma>n}^{i}$ is dense in $[p]$.

(4) $U_{\sigma}^{j}=U_{\sigma}^{i}$ (and thus $V_{\sigma}^{j}=V_{\sigma}^{i}$ ).

Note that the definition of the $V_{\sigma}^{i}$ in terms of $U_{\sigma}^{i}$ guarantees that $U_{\sigma}^{i} \cup V_{\sigma}^{i}$ is dense and $U_{\sigma}^{i} \cap V_{\sigma}^{i}=\emptyset$, and the remaining parts of the claim suffice to establish that we have a Baire decomposition.

When $\sigma$ is a leaf, it is clear that $U_{\sigma}^{i}$ and $U_{\sigma}^{j}$ consist of precisely those $p$ such that $[p]$ is contained in the clopen set coded by $\ell(\sigma)$.

Now fix an interior node $\sigma$. By induction, we can assume that for all $\tau \in T$ properly extending $\sigma$, condition (4) holds, so we drop the superscripts and denote these open sets by $U_{\tau}$ and $V_{\tau}$. Since Properties (1)-(3) hold for $\rho$ extending such $\tau$, we have that $\left(U_{\rho}, V_{\rho}\right)_{\rho \in T_{\tau}}$ are a Baire decomposition for $T_{\tau}$. We let $D_{m, \tau}$ denote the canonical sequence of dense open sets from Proposition 3.9 corresponding to this Baire decomposition. Since $\left(D_{m, \tau}\right)_{m} \in \Delta_{1}^{1}\left(G_{i}\right) \cap \Delta_{1}^{1}\left(G_{j}\right)$, so by Proposition 2.20. $\left(D_{m, \tau}\right)_{m} \in \Delta_{1}^{1}$. Therefore, for all $p \in 2^{<\omega}$, we have $p^{\wedge} G_{i}, p^{\frown} G_{j} \in \cap_{m} D_{m, \tau}$. Therefore, if $p^{\frown} G_{i} \in U_{\tau}$, then $p^{\frown} G_{i} \in\left|T_{\tau}\right|$, and if $p^{\frown} G_{i} \in V_{\tau}$, then $p^{\frown} G_{i} \notin\left|T_{\tau}\right|$, and the same holds for $G_{j}$.

Suppose that $\sigma$ is a union node. To prove $(\Rightarrow)$ in (2), fix $q \in U_{\sigma}^{i}$. We need to show that $\left\{r \in 2^{<\omega}: q^{\wedge} r \in \bigcup_{n} U_{\sigma^{\wedge} n}\right\}$ is dense. For a contradiction, suppose $\left[q^{\wedge} r_{0}\right] \cap \bigcup_{n} U_{\sigma \wedge n}=\emptyset$ for some fixed $r_{0}$. To obtain a contradiction, we will show that for all $n$, we have $q^{\frown} r_{0}^{\frown} G_{i} \notin\left|T_{\sigma^{\wedge} n}\right|$. Since $\sigma$ is a union node, it follows that $q^{\wedge} r_{0}^{\frown} G_{i} \notin\left|T_{\sigma}\right|$ contradicting the fact that $q \in U_{\sigma}^{i}$.

Fix $n$ and let $\tau=\sigma^{\wedge} n$. Since $\tau$ properly extends $\sigma$, we have that $q^{\wedge} r_{0}^{\frown} G_{i} \in$ $\bigcap_{m} D_{m, \tau}$ by the comments two paragraphs above. Since $U_{\tau} \cup V_{\tau}$ is dense but $U_{\tau} \cap\left[q^{\frown} r_{0}\right]=\emptyset$, it follows that $V_{\tau}$ is dense in $\left[q^{\frown} r_{0}\right]$ and therefore $q^{\frown} r_{0}^{\frown} G_{i} \in V_{\tau}$. From $q^{\frown} r_{0}^{\frown} G_{i} \in \bigcap_{m} D_{m, \tau}$ and $q^{\frown} r_{0}^{\frown} G_{i} \in V_{\tau}$, it follows that $q^{\frown} r_{0}^{\frown} G_{i} \notin\left|T_{\tau}\right|$ as required to complete the contradiction.

To prove $(\Leftarrow)$ in $(2)$, assume that $\bigcup_{n} U_{\sigma^{-n} n}$ is dense in $[q]$. We need to show that $q \in U_{\sigma}^{i}$. Fix $r_{0} \in 2^{<\omega}$. Since $\bigcup_{n} U_{\sigma\urcorner n}$ is dense in [q], it is also dense in $\left[q^{\wedge} r_{0}\right]$. By the induction hypothesis and Proposition 2.20, $\bigcup_{n} U_{\sigma^{\wedge} n}$ is $\Delta_{1}^{1}$. Let $A=\{\tau$ : $\left.\exists n\left(q^{\wedge} r_{0}^{\frown} \tau \in U_{\sigma^{\wedge} n}\right)\right\}$. $A$ is dense and is $\Delta_{1}^{1}$. Therefore, $G_{i}$ meets the set $A$. Fix $\tau \in A$ such that $\tau \prec G_{i}$ and fix $n$ such that $q^{\wedge} r_{0}^{\curvearrowright} \tau \in U_{\sigma \wedge n}$. Then $q^{\wedge} r_{0}^{\frown} G_{i} \in U_{\sigma\urcorner n}$. So, as noted above, $q^{\curvearrowright} r_{0}^{\frown} G_{i} \in \bigcap_{m} D_{m, \sigma^{\curvearrowright} n}$ and so $q^{\frown} r_{0}^{\frown} G_{i} \in\left|T_{\sigma \frown n}\right|$. As $r_{0}$ was arbitrary, this shows that $q \in U_{\sigma}^{i}$. 
The exact same argument shows that (2) is also satisfied when $i$ is replaced by $j$. Therefore, $U_{\sigma}^{i}$ and $U_{\sigma}^{j}$ are described by exactly the same condition, so they are equal.

Finally, let $\sigma$ be an intersection node. First, consider the direction $(\Leftarrow)$ of $(3)$ : Suppose that $q \notin V_{\sigma}^{i}$ and fix $r_{0}$ such that $q^{\frown} r_{0} \in U_{\sigma}^{i}$. We will show that $q^{\frown} r_{0} \in U_{\sigma^{\wedge} n}$ for all $n$, so $\bigcup_{n} V_{\sigma \sim n}$ is not dense in $[q]$ (it is disjoint from $\left[q^{\wedge} r_{0}\right]$ ).

Fixing $n$, consider an arbitrary string $p$ extending $q^{\frown} r_{0}$. Since $q^{\frown} r_{0} \in U_{\sigma}^{i}$, we know that $p^{\frown} G_{i} \in\left|T_{\sigma}\right|$. Since $\sigma$ is an intersection node, it follows that $p^{\frown} G_{i} \in$ $\left|T_{\sigma \frown n}\right|$. Since $p$ was an arbitrary string extending $q^{\frown} r_{0}$, this implies $q^{\frown} r_{0} \in U_{\sigma \frown n}$ as required to complete this direction of (3).

To prove $(\Rightarrow)$ in (3), assume $\bigcup_{n} V_{\sigma \sim n}$ is not dense in $[q]$. We need to show that $q \notin V_{\sigma}^{i}$. Fix $r_{0}$ such that $\left[q^{\wedge} r_{0}\right] \cap \bigcup_{n} V_{\sigma^{\wedge} n}=\emptyset$. Therefore, for each $n, U_{\sigma^{\wedge} n}$ is dense in $\left[q^{\curvearrowleft} r_{0}\right]$.

Fix an arbitrary string $p$ extending $q^{\curvearrowright} r_{0}$. We claim that for all $n$, we have $p^{\curvearrowleft} G_{i} \in$ $U_{\sigma \frown n}$. First, note that $U_{\sigma \wedge n}$ is dense in $[p]$ and that by the induction hypothesis and Proposition 2.20, $U_{\sigma^{\wedge} n}$ is $\Delta_{1}^{1}$. We shift $U_{\sigma^{\wedge} n}$ to a set $A=\left\{\tau: p^{\wedge} \tau \in U_{\sigma^{\wedge} n}\right\}$ which is dense and $\Delta_{1}^{1}$, so $G_{i}$ meets $A$. Let $\tau \in A$ be such that $\tau \prec G_{i}$. Then, $p^{\wedge} \tau \in U_{\sigma \frown n}$ and so $p^{\frown} G_{i} \in U_{\sigma\urcorner n}$. Furthermore, as noted above, since $p^{\frown} G_{i} \in \bigcap_{m} D_{m, \sigma\urcorner n}$, it follows that $p^{\wedge} G_{i} \in\left|T_{\sigma \sim n}\right|$. Since this property holds for each $n$ and since $\sigma$ is an intersection node, it follows that $p^{\frown} G_{i} \in\left|T_{\sigma}\right|$. The string $p$ extending $q^{\frown} r_{0}$ was arbitrary, so by the definition of $U_{\sigma}^{i}$, we have $q^{\curvearrowleft} r_{0} \in U_{\sigma}^{i}$, and therefore $q \notin V_{\sigma}^{i}$ to complete the proof of (3).

We have actually proved a little more. Inspecting the argument for $(\Rightarrow)$ in (3), we see that whenever $[q] \cap \bigcup_{n} V_{\sigma^{\wedge} n}=\emptyset$, we have $q \in U_{\sigma}^{i}$; and inspecting the argument for $(\Leftarrow)$ in $(3)$, we see that whenever $q \in U_{\sigma}^{i}$, we have $[q] \cap \bigcup_{n} V_{\sigma \frown n}=\emptyset$. This gives a definition of $U_{\sigma}^{i}$ that does not depend on $i$, and indeed the arguments above could be repeated exactly for $U_{\sigma}^{j}$. Therefore, $U_{\sigma}^{i}=U_{\sigma}^{j}$ in the case where $\sigma$ is an intersection as well.

We conclude that $\left(U_{\sigma}, V_{\sigma}\right)_{\sigma \in T}$ is a Baire decomposition for $T$, and so $T$ has a Baire approximation in $M$. Therefore $\mathcal{M}$ satisfies CD-PB but not ATR .

\section{Decorating trees}

In order to show that CD-PB is strictly stronger than $L_{\omega_{1}, \omega}-C A$, we need to make some techniques for building non-standard Borel codes in a way that ensures they are completely determined.

A non-standard Borel code is a code that is not actually well-founded, but which the model thinks is well-founded. These fake codes are essential for the strength of CD-PB. If a Borel code is truly well-founded, then it has a Baire code which is hyperarithmetic in itself. Since any $\omega$-model of $L_{\omega_{1}, \omega}$-CA is closed under hyperarithmetic reduction, $\mathrm{L}_{\omega_{1}, \omega}$-CA alone would be enough to guarantee the Baire code exists in the case when the Borel code is truly well-founded (at least in $\omega$-models). So now we are going to describe how to construct a non-standard Borel code which makes every effort to be completely determined.

If we make a Borel code $T$ which is not well-founded, the most likely scenario is that it is also not completely determined. This is because, in general, it might take a jump hierarchy the height of the rank of $T$ in order to produce an evaluation map. So in this section, we show how to add "decorations" to the tree, which shortcut the logic of the tree to make sure that for a small set of $X$, there is an evaluation 
map for $X$ in the decorated tree. In Section 6. "small" is countable, and in Section 7. "small" is meager. This comes at the cost of trashing any information about whether $X$ was in the original set, but if that set had a Baire approximation, then its decorated version should have the same Baire approximation, since the set of $X$ whose membership facts were overwritten is small. We use this to show that if the model satisfies CD-PB, then the "small" set cannot be the entire second-order part of the model.

Suppose that we have a partial computable function $h$ which maps a number $b \in \mathcal{O}^{*}$ to a pair of $b$-ranked labeled trees $\left(P_{b}, N_{b}\right)$. We do not mind if $h$ happens to also make some outputs for $b \notin \mathcal{O}^{*}$.

The intention is that when $b \in \mathcal{O}$, any $X \in\left|P_{b}\right| \cup\left|N_{b}\right|$ will have an approximately $H_{b}^{X}$-computable evaluation map in the decorated tree, and $X$ will be in the decorated tree if $X \in\left|P_{b}\right|$ and out of the decorated tree if $X \in\left|N_{b}\right|$. (In practice we will always have $\left|P_{b}\right| \cap\left|N_{b}\right|=\emptyset$.)

The operation Decorate is defined below using effective transfinite recursion (with parameter $<_{*}$; see comment in the next paragraph), and therefore is welldefined on $a$-ranked trees $T$ for all $a \in \mathcal{O}^{*, T}$. This is because the effective transfinite recursion can be carried out in $H Y P(T)$ with the same result.

Note that as it is defined here, Decorate is not quite a computable operation. That is because the relation $<_{*}$ is only c.e., not computable. To make Decorate computable, one should replace $\langle 2 b+1\rangle$ below with $\langle 2\langle b, s\rangle+1\rangle$, where $s$ is the stage at which we learn that $b<_{*} \rho_{T}(\lambda)$. This has no effect on the logic of the tree, but does result in excessive notational clutter. The reader who prefers a computable operation could replace $\langle 2 b+1\rangle$ everywhere with the more complicated expression above. For our purposes, it is perfectly fine that Decorate is computable relative to the parameter $<_{*}$ (a linear order which is itself $\emptyset^{\prime}$-computable). In any case, all results of this section do relativize and they will later be used in a relativized form.

Definition 5.1. The operation Decorate is defined as follows. The inputs are an a-ranked labeled tree $T$ and a partial computable function $h$ as above.

$$
\begin{aligned}
\operatorname{Decorate}(T, h)=\{\lambda\} & \left.\cup \bigcup_{\langle n\rangle \in T}\langle 2 n\rangle\right\urcorner \operatorname{Decorate}\left(T_{\langle n\rangle}, h\right) \\
& \cup \bigcup_{b<_{*} \rho_{T}(\lambda)}\langle 2 b+1\rangle \frown \operatorname{Decorate}\left(Q_{b}, h\right)
\end{aligned}
$$

where $Q_{b}=P_{b}$ if $\lambda$ is $a \cup$ in $T$, and $Q_{b}=N_{b}^{c}$ if $\lambda$ is $a \cap$ in $T$.

The rank and label of $\lambda$ in Decorate $(T, h)$ are defined to coincide with the rank and label of $\lambda$ in $T$. The ranks and labels of the other nodes in Decorate $(T, h)$ are inherited from Decorate $\left(T_{\langle n\rangle}, h\right)$ or Decorate $\left(Q_{b}, h\right)$ as appropriate.

Since $P_{b}$ and $N_{b}$ are $b$-ranked, Decorate $(T, h)$ satisfies the local requirements on a ranking. So if $T$ is $a$-ranked, so is $\operatorname{Decorate}(T, h)$.

Similarly, if $T$ and each $P_{b}$ and $N_{b}$ are alternating, and each $P_{b}$ and $N_{b}$ have an intersection or leaf at their root, then $\operatorname{Decorate}(T, h)$ will also be alternating. (Note that in this case, $N_{b}^{c}$ has a union at its root).

The following is the essential feature of a decorated tree.

Proposition 5.2. If $\sigma \in \operatorname{Decorate}(T, h)$ has rank $b$, then for all $d<_{*} b$,

$$
\operatorname{Decorate}(T, h)_{\sigma^{\frown}\langle 2 d+1\rangle}=\operatorname{Decorate}\left(Q_{d}, h\right),
$$


where $Q_{d}=P_{d}$ or $N_{d}^{c}$ as appropriate.

Proof. By induction on the length of $\sigma$, if $\sigma \in \operatorname{Decorate}(T, h)$, then there is some tree $S$ such that Decorate $(T, h)_{\sigma}=\operatorname{Decorate}(S, h)$. The rank of $\sigma$ in Decorate $(T, h)$ coincides with the rank of $\lambda$ in $S$, and this rank is $b$. Therefore, by the definition of Decorate $(S, h)$, we have $\operatorname{Decorate}(S, h)_{\langle 2 d+1\rangle}=\operatorname{Decorate}\left(Q_{d}, h\right)$.

Definition 5.3. A nice decoration generator is a partial computable function which maps any $b \in \mathcal{O}^{*}$ to alternating, $b$-ranked trees $\left(P_{b}, N_{b}\right)$, where each $P_{b}$ and $N_{b}$ have an intersection or a leaf at their root.

Lemma 5.4. Let $h$ be a nice decoration generator. Suppose $b \in \mathcal{O}$, and suppose that $X \notin\left|P_{d}\right| \cup\left|N_{d}\right|$ for any $d<_{*} b$. Then for any $b$-ranked tree $T, X \in|\operatorname{Decorate}(T, h)|$ if and only if $X \in|T|$.

Proof. By induction on $b$. Since $b \in \mathcal{O}, T$ is truly well-founded, so there is a unique evaluation map $f$ for $X$ in $T$. Further, for each $d<_{*} b$, there are unique evaluation maps $g_{P, d}, g_{N, d}$ for $X$ in Decorate $\left(P_{d}, h\right)$ and Decorate $\left(N_{d}^{c}, h\right)$. Consider the function $g$ : Decorate $(T, h) \rightarrow\{0,1\}$ defined by

$$
g(\sigma)= \begin{cases}f\left(\frac{\sigma}{2}\right) & \text { if each component of } \sigma \text { is even } \\ g_{Q, d}\left(\sigma_{1}\right) & \text { if } \sigma=\sigma_{0}\langle 2 d+1\rangle \frown \sigma_{1} \text { and each component of } \sigma_{0} \text { is even, }\end{cases}
$$

where the division $\sigma / 2$ is taken componentwise, and where $Q$ is either $P$ or $N$ depending on whether $\sigma_{0}$ is a union or intersection in $\operatorname{Decorate}(T, h)$.

Since $g(\lambda)=f(\lambda)$, it is enough to show that $g$ is an evaluation map for $X$ in Decorate $(T, h)$. Clearly $g$ satisfies the logic of the tree at leaves and at nodes which have an odd component. Consider $\sigma \in \operatorname{Decorate}(T, h)$ where $\sigma$ is a $\cup$ and all components of $\sigma$ are even. By induction, since $P_{d}$ is a $d$-ranked tree, $X \in$ $\left|\operatorname{Decorate}\left(P_{d}, h\right)\right|$ if and only if $X \in\left|P_{d}\right|$. By hypothesis, $X \notin\left|P_{d}\right|$, so $g_{P, d}(\lambda)=0$, so by Proposition 5.2, $g\left(\sigma^{\curvearrowright}\langle 2 d+1\rangle\right)=0$. Therefore, the nodes of this form can be ignored: we have

$$
\exists m\left(g\left(\sigma^{\frown} m\right)=1\right) \Longleftrightarrow \exists n\left(g\left(\sigma^{\curvearrowright}\langle 2 n\rangle\right)=1\right) \Longleftrightarrow f(\sigma / 2)=1
$$

so $g(\sigma)$ takes the correct value. The argument if $\sigma$ is a $\cap$ is similar, except that as $X \notin\left|N_{b}\right|$, we have $X \in\left|N_{b}^{c}\right|$, and therefore $g_{N, d}(\lambda)=1$, meaning that nodes of the form $\sigma^{\wedge}\langle 2 d+1\rangle$ can be safely ignored when taking an intersection.

Lemma 5.5. Let $a \in \mathcal{O}^{*}$ and $b \in \mathcal{O}$ with $b<_{*} a$. Let $T$ be an alternating, a-ranked tree and let $h$ be a nice decoration generator. Suppose $X \in\left|P_{b}\right| \cup\left|N_{b}\right|$. Then

(1) $X$ has a unique evaluation map in $\operatorname{Decorate}(T, h)$.

(2) This evaluation map is $H_{b+O(1)}^{X \oplus T}$-computable.

(3) If $b$ is $<_{*}$-minimal such that $X \in\left|P_{b}\right| \cup\left|N_{b}\right|$, and $b<_{*} \rho_{T}(\langle n\rangle)$ for all $\langle n\rangle \in T$, and $g$ is the unique evaluation map for $X$ in Decorate $(T, h)$, then

(a) $X \in\left|P_{b}\right| \backslash\left|N_{b}\right| \Longrightarrow g(\lambda)=1$

(b) $X \in\left|N_{b}\right| \backslash\left|P_{b}\right| \Longrightarrow g(\lambda)=0$.

Proof. It suffices to show all three parts in the case when $b$ is $<_{*}$-minimal such that $X \in\left|P_{b}\right| \cup\left|N_{b}\right|$.

We prove (1) and (2) by showing that for each $\sigma \in \operatorname{Decorate}(T, h)$, there is only one possible value for $g(\sigma)$ for any evaluation map $g$ for $X$ in $\operatorname{Decorate}(T, h)$ and that $H_{b+O(1)}^{X \oplus T}$ suffices to compute this value. Since these unique values satisfy the 
internal logic of the tree (which the reader can verify from the description below), they constitute an evaluation function for $X$ in Decorate $(T, h)$, proving (1) and (2).

To show that there is only one possible value for $g(\sigma)$, we break into cases depending on the rank and label of $\sigma$ in $\operatorname{Decorate}(T, h)$ and on whether $X \in\left|P_{b}\right|$ or $X \in\left|N_{b}\right|$. Note that $H_{b}^{X \oplus T}$ can uniformly determine the appropriate case for each $\sigma$.

Case 1. Suppose $\rho(\sigma) \leq_{*} b$. Since $b \in \mathcal{O}$, Decorate $(T, h)_{\sigma}$ is truly well-founded. Therefore, there is a unique evaluation map $f$ for $X$ in Decorate $(T, h)_{\sigma}$ and we have $g(\sigma)=f(\lambda)$. The map $f$ is uniformly $H_{b+O(1)}^{X \oplus T}$-computable.

Case 2. Suppose $b<_{*} \rho(\sigma), \sigma$ is a union node in Decorate $(T, h)$ and $X \in\left|P_{b}\right|$. In this case, we claim that $g(\sigma)=1$. By Proposition 4.2, all nodes extending $\sigma^{\frown}\langle 2 b+1\rangle$ have rank $b$ or less. Therefore, there is a unique evaluation map $f$ on Decorate $(T, h)_{\sigma^{\sim}\langle 2 b+1\rangle}$ and so $g\left(\sigma^{\frown}\langle 2 b+1\rangle\right)=f(\lambda)$. By Lemma 4.4, $X \in\left|P_{b}\right|$ implies $f(\lambda)=1$. Therefore, $g\left(\sigma^{\curvearrowright}\langle 2 b+1\rangle\right)=1$ and because $\sigma$ is a union node, $g(\sigma)=1$.

Case 3. Suppose $b<_{*} \rho(\sigma), \sigma$ is an intersection node in Decorate $(T, h)$ and $X \in\left|P_{b}\right|$. Since Decorate $(T, h)$ is alternating, each node $\sigma^{\curvearrowright} m$ is either a union node or a leaf. If $\rho\left(\sigma^{\wedge} m\right) \leq_{*} b$, then the value of $g\left(\sigma^{\wedge} m\right)$ is fixed as in Case 1 . If $b<_{*} \rho\left(\sigma^{\curvearrowright} m\right)$, then $g\left(\sigma^{\wedge} m\right)=1$ as in Case 2. Together, these values determine $g(\sigma)$ uniquely. $H_{b}^{X \oplus T}$ suffices to compute the values of $g\left(\sigma^{\wedge} m\right)$ and it takes one extra jump to determine if $g\left(\sigma^{\wedge} m\right)=1$ for all $m$, and hence determine $g(\sigma)$.

Case 4. Suppose $b<_{*} \rho(\sigma), \sigma$ is an intersection node in Decorate $(T, h)$ and $X \in\left|N_{b}\right|$. An analogous argument to Case 2 shows that $g(\sigma)=0$.

Case 5. Suppose $b<_{*} \rho(\sigma), \sigma$ is a union node in Decorate $(T, h)$ and $X \in\left|N_{b}\right|$. This case is analogous to Case 3 and the unique value of $g(\sigma)$ can be determined with one extra jump.

These cases are exhaustive, but if $\left|P_{b}\right| \cap\left|N_{b}\right| \neq \emptyset$, then more than one case can apply. However, if $X \in\left|P_{b}\right| \cap\left|N_{b}\right|$, the cases are compatible. In this degenerate situation, we have that for any $\sigma$ such that $b<_{*} \rho(\sigma), g(\sigma)=1$ if $\sigma$ is a union node and $g(\sigma)=0$ if $\sigma$ is an intersection node. This completes the proof of (1) and (2).

For (3), if $X \in\left|P_{b}\right| \backslash\left|N_{b}\right|$, and if $\lambda$ is $\cup$, then $g(\lambda)=1$ just as above. But if $\lambda$ is $\cap$, then we claim that for each $m, g(\langle m\rangle)=1$. (Note that neither $\lambda$ nor $\langle m\rangle$ can be a leaf in $T$ because $b<_{*} a$ and the hypothesis on part (3) specifies that $b<_{*} \rho_{T}(\langle m\rangle)$ for each $m$ ). If $m=2 n$ for some $\langle n\rangle \in T$, or if $m=2 d+1$ for some $d>_{*} b$, then because $b<_{*} \rho_{T}(\langle n\rangle)$ for all $n$, and each $\langle m\rangle$ is a union, again we have $g(\langle m\rangle)=1$ for such $m$. In the remaining case, when $m=2 d+1$ with $d \leq_{*} b$, then since $b$ is minimal such that $X \in\left|P_{b}\right| \cup\left|N_{b}\right|$, and $X \notin\left|N_{b}\right|$, we have $X \in\left|N_{d}^{c}\right|$. So by Lemma 5.4, $X \in\left|\operatorname{Decorate}\left(N_{d}^{c}, h\right)\right|$, so $g(\langle 2 d+1\rangle)=1$. Since $g(\langle m\rangle)=1$ for all $m$, we have $g(\lambda)=1$ as well. A complementary argument establishes (3b).

\section{CD-PB DOES NOT HOLD IN HYP}

We now show that CD-PB is not a theory of hyperarithmetic analysis by showing that CD-PB fails in the $\omega$-model $H Y P$. In brief, we let $E_{a}$ code a canonical universal $\Sigma_{a}^{0}$ set. Applying this definition also to pseudo-ordinals $a^{*}$, we make a computable code for the set

$$
\bigcup_{b<a_{*} a^{*}}\left|E_{b}\right| \cap\left\{X: b \text { is least s.t. } X \leq_{T} H_{b}\right\}
$$


We decorate the code to give each $H_{b}$-computable set an $H_{b}$-computable evaluation map. Then we argue that the result is a code which $H Y P$ thinks is well-founded and completely determined, but which can have no $H Y P$ Baire approximation.

Theorem 6.1. CD-PB does not hold in HYP.

Proof. Using Proposition 2.1, there is a computable procedure which, on inputs $a \in \mathcal{O}, e \in \mathbb{N}, p \in 2^{<\omega}$, outputs an index for a $2^{a}$-ranked computable $L_{\omega_{1}, \omega}$ formula $F_{a, e, p}$, which holds true if and only if $p \in W_{e}^{H_{a}}$. Transform each formula $F_{a, e, p}$ into a Borel code by swapping false for $\emptyset$, and true for $\left[0^{e} 1^{\frown} p\right]$. Then take the union of all of these, obtaining a code $E_{a}$ of rank $a+O(1)$ such that for all $a \in \mathcal{O}$,

$$
\left|E_{a}\right|=\bigcup_{e, p: p \in W_{e}^{H_{a}}}\left[0^{e} 1^{\frown} p\right] .
$$

For any pseudo-ordinal $a^{*}, W_{p\left(a^{*}\right)}$ is not well-founded, but it has no hyperarithemtic descending sequence, so $H Y P$ believes $W_{p\left(a^{*}\right)}$ is well-founded. Then $H Y P$ also believes that $E_{b}$ is well-founded for any $b<_{*} a^{*}$, because $E_{b}$ is $(b+O(1))$-ranked, so any path through $E_{b}$ would reveal a descending sequence in $W_{p\left(a^{*}\right)}$. We may assume that $E_{b}$ are alternating and $(b+O(1))$-ranked for all $b \leq_{*} a^{*}$. For the sake of a later application of Lemma [5.5. note that we can also assume that the rank of $E_{a^{*}}$ is a successor, so of the form $2^{x}$ for some $x$, and that for each $\langle n\rangle \in E_{a^{*}}$, the rank of $\langle n\rangle$ in $E_{a^{*}}$ is $x$.

Similarly, there is a computable procedure which, for each $b \in \mathcal{O}$, outputs a $(b+O(1))$-ranked Borel code $S_{b}$ such that

$$
\left|S_{b}\right|=\left\{X \in 2^{\omega}: X \leq_{T} H_{b} \text { and for all } c<_{*} b, X \not_{T} H_{c}\right\} .
$$

We think of $S_{b}$ as coding a slice of $H Y P$. Just as for $E_{b}$, we have that for any $b<_{*} a^{*}, H Y P$ thinks that $S_{b}$ is well-founded.

For each $b<_{*} a^{*}$, define $P^{b}$ and $N^{b}$ so that they are alternating, and

$$
\left|P^{b}\right|=\left|S_{b}\right| \cap\left|E_{b}\right|, \quad\left|N^{b}\right|=\left|S_{b}\right| \cap\left|E_{b}^{c}\right| .
$$

Observe that $P^{b}$ and $N^{b}$ can be both $(b+k)$-ranked, where $k$ is some fixed finite ordinal. Let $h$ be the function which, on input $b$, outputs $P_{b}=P^{b-k}$ and $N_{b}=N^{b-k}$ if the operation $b-k$ can be performed, and outputs a degenerate $b$-ranked tree coding the empty set, if $b$ is less than $k$ successors from a limit ordinal.

We claim that Decorate $\left(E_{a^{*}}, h\right)$ is completely determined in $H Y P$. Observe that $h$ is a nice decoration generator. Let $X \in H Y P$. Then there is some $b \in \mathcal{O}$ with $b<_{*} a$ such that $X \leq_{T} H_{b}$. Since $a^{*}$ is a pseudo-ordinal, $b+O(1)<_{*} a^{*}$ is satisfied. By the choice of $b$ we have $X \in\left|S_{b}\right|=\left|P_{b+k}\right| \cup\left|N_{b+k}\right|$. Therefore, by Lemma 5.5, $X$ has a $H Y P$ evaluation map. Therefore, Decorate $\left(E_{a^{*}}, h\right)$ is completely determined in $H Y P$.

Suppose for contradiction that Decorate $\left(E_{a^{*}}, h\right)$ has a $H Y P$ Baire approximation. Let $b \in \mathcal{O}$ with $b<_{*} a^{*}$ and with the Baire approximation $\left(U, V,\left\{D_{n}\right\}_{n \in \omega}\right) \leq_{T}$ $H_{b}$. By the recursion theorem, there is an index $e$ such that

$$
W_{e}^{H_{b}}=\left\{p: 0^{e} 1^{\frown} p \in V\right\}
$$

where $H_{b}$ is used to compute $V$. Choose $p$ with $0^{e} 1^{\frown} p \in U \cup V$, this is possible as $U \cup V$ is dense. Let $X \in H Y P$ be such that

(1) $0^{e} 1^{\frown} p \prec X$

(2) $X \leq_{T} H_{b}$ but $X \not_{T} H_{c}$ for any $c<_{*} b$, 
(3) $X \in D_{n}$ for all $n$.

This is possible because the $D_{n}$, and the dense sets which need to be met to avoid being computed by $H_{c}$ for $c<_{*} b$, are uniformly $H_{b}$-computable.

Now $b+k$ is least such that $X \in\left|P_{b+k}\right| \cup\left|N_{b+k}\right|=\left|S_{b}\right|$. By Lemma 5.5, $X \in\left|\operatorname{Decorate}\left(E_{a^{*}}, h\right)\right|$ if and only if $X \in\left|E_{b}\right|$. Because $X$ meets each $D_{n}$ and $U \cup V$, by the definition of a Baire code, we have $X \in\left|\operatorname{Decorate}\left(E_{a^{*}}, h\right)\right|$ if and only if $X \in U$. To establish the contradiction, it suffices to show that $X \in\left|E_{b}\right|$ if and only if $X \in V$.

Observe $X \in\left|E_{b}\right|$, if and only if, for some $q$ extending $p$, we have $0^{e} 1^{\frown} q \prec X$ and $q \in W_{e}^{H_{b}}$. But this happens if and only if for some such $q$, we have $0^{e} 1^{\frown} q \in V$.

\section{CD-PB IMPLIES $H Y P$ GENERICS EXIST IN $\omega$-MODELS}

The next theorem shows that CD-PB implies the existence of hyperarithmetic generics in $\omega$-models. In short, if $\mathcal{M}$ has $Z$ but no $\Delta_{1}^{1}(Z)$-generics, there is a pseudo-ordinal $a^{*}$ which $\mathcal{M}$ thinks is well-founded. This pseudo-ordinal can be used to construct a code for the following subset of $M$, where $E_{b}$ denotes a code for a universal $\Sigma_{b}^{Z}$ set:

$$
\bigcup_{b<* a^{*}}\left|E_{b}\right| \cap\left\{X: b \text { is least s.t. } X \text { is not generic relative to } H_{b}^{Z}\right\}
$$

After decorating this code, it becomes completely determined for every non- $\Delta_{1}^{1}(Z)$ generic. If this code has a Baire decomposition, meeting the associated dense sets creates a $\Delta_{1}^{1}(Z)$-generic.

Theorem 7.1. If $\mathcal{M}$ is an $\omega$-model which satisfies $\mathrm{CD}-\mathrm{PB}$, then for every $Z \in \mathcal{M}$, there is a $G \in \mathcal{M}$ such that $G$ is $\Delta_{1}^{1}$-generic relative to $Z$.

Proof. Let $M$ be the second-order part of an $\omega$-model which satisfies CD-PB. Then by Proposition 3.6, whenever $Z \in M$, we also have that $H_{b}^{Z} \in M$ for every $b \in \mathcal{O}^{Z}$.

Case 1: Suppose $\mathcal{M}$ is a $\beta$-model (that is, for every tree $T \in M$, if $\mathcal{M} \models$ " $T$ is well-founded", then $T$ is truly well-founded.) Let $Z \in M$. Because $\{G$ : $G$ is $\Delta_{1}^{1}(Z)$-generic $\}$ is a $\Sigma_{1}^{1}(Z)$ set, the $Z$-computable tree corresponding to the $\Sigma_{1}^{1}(Z)$ statement "there is a $\Delta_{1}^{1}(Z)$-generic" has a path in $M$, and that path computes a $\Delta_{1}^{1}(Z)$-generic $G$. Therefore, the theorem holds when $\mathcal{M}$ is a $\beta$-model.

Case 2: Suppose that there is some tree $S \in M$ which $\mathcal{M}$ believes is well-founded, but in reality is ill-founded. Let $Z \in M$, and without loss of generality assume that $Z \geq_{T} S$ (without this assumption we find a $\Delta_{1}^{1}(Z \oplus S)$-generic $G$, but such $G$ is also $\Delta_{1}^{1}(Z)$-generic.) By Proposition 2.2 , there is a $Z$-computable function which, given the index of a truly well-founded $Z$-computable linear order, outputs an element of $\mathcal{O}^{Z}$ which bounds its order type. Applying that function to the Kleene-Brouwer ordering on $S$ produces a pseudo-ordinal $a^{*} \in \mathcal{O}^{*, Z}$ such that $W_{p\left(a^{*}\right)}^{Z}$ is not truly well-founded, but it has no descending sequence in $M$.

Relativize the definitions of $<_{*}$, ranked trees, Decorate, and Lemmas 5.4 and 5.5 to $Z$. Note that because $M$ is hyperarithmetically closed, all the evaluation maps provided by relativized versions of Lemmas 5.4 and 5.5 are in $M$.

As in the previous theorem, there is a $Z$-computable procedure which maps any $b \in \mathcal{O}^{Z}$ to an alternating code $E_{b}$ of rank $b+O(1)$ such that

$$
\left|E_{b}\right|=\bigcup_{e, r: r \in W_{e}^{H_{b}^{Z}}}\left[0^{e} 1^{\frown} r\right] .
$$


Further, using Proposition 2.1 there are $Z$-computable procedures which map each $b \in \mathcal{O}^{Z}$ to a code $S_{b}$ of rank $b+O(1)$ such that

$$
\begin{aligned}
\left|S_{b}\right|=\left\{X \in 2^{\omega}: X \text { is not } 1 \text {-generic relative to } H_{b}^{Z},\right. \\
\text { but for all } \left.c<_{*}^{Z} b, X \text { is 1-generic relative to } H_{c}^{Z}\right\},
\end{aligned}
$$

and alternating codes $P_{b}$ and $N_{b}$ of rank $b$ such that

$$
\left|P_{b}\right|=\left|S_{b-O(1)}\right| \cap\left|E_{b-O(1)}\right|, \quad\left|N_{b}\right|=\left|S_{b-O(1)}\right| \cap\left|E_{b-O(1)}^{c}\right|,
$$

(and for $b$ that are within $O(1)$ of a limit ordinal, $P_{b}$ and $N_{b}$ are degenerate $b$-ranked trees coding the empty set as before).

Let us be a little more specific and say that the code for $P_{b}$ is made exactly as one would expect: it is $\operatorname{Alternate}\left(P_{b}^{\prime}\right)$, where

$$
P_{b}^{\prime}=\{\lambda\} \cup\langle 0\rangle^{\frown} S_{b-O(1)} \cup\langle 1\rangle^{\frown} E_{b-O(1)},
$$

the root $\lambda$ is a $\cap$ of rank $b$ in $P_{b}^{\prime}$, and all other ranks and labels are inherited from their respective subtrees. We remark that because the root of $P_{b}^{\prime}$ is a $\cap$, the root of $E_{b+O(1)}$ is a $\cup$, and $E_{b+O(1)}$ is already alternating, we have $\left(P_{b}\right)_{\langle 1\rangle}=E_{b-O(1)}$.

Because the outputs of Proposition 2.1 are well-defined for all $b \in \mathcal{O}^{Z}$, so also are the codes $P_{b}$ and $N_{b}$. Also, for any $b<_{*}^{Z} a^{*}, M$ believes these codes to be well-founded because they are $b$-ranked.

Let $h$ be the name of the nice decorating function mapping $b$ to $\left(P_{b}, N_{b}\right)$, and consider the code $T:=\operatorname{Decorate}^{Z}\left(E_{a^{*}}, h\right)$. Observe that since $\lambda$ in $E_{a^{*}}$ is a $\cup$, we know that $\lambda$ in $T$ is a $\cup$.

If $T$ is not completely determined, let $G \in M$ be such that $G$ does not have an evaluation map in $T$. We claim that $G$ is $\Delta_{1}^{1}(Z)$-generic. If $G$ is not $\Delta_{1}^{1}(Z)$-generic, then there is some least $b \in \mathcal{O}^{Z}$ with $b<_{*}^{Z} a^{*}$ such that $G$ is not 1-generic relative to $H_{b}^{Z}$. Then we would have $G \in\left|S_{b}\right|$, and therefore by Lemma [5.5, $G$ would have an evaluation map in $T$.

If $T$ is completely determined, then since $\mathcal{M}$ models CD-PB, let $\left(U_{\sigma}, V_{\sigma}\right)_{\sigma \in T} \in M$ be a Baire decomposition for $T$. Let $\left\{D_{i}\right\}_{i<\omega} \in M$ be the associated sequence of dense sets as in Proposition 3.9. For any $p \in 2^{<\omega}$, define $D_{i, p}=\left\{q: p^{\curlyvee} q \in D_{i}\right\}$. We claim that any $G \in \cap_{i, p} D_{i, p}$ is $\Delta_{1}^{1}(Z)$-generic. For this we argue that every dense open $B \in \Delta_{1}^{1}(Z)$ actually contains $D_{i, p}$ for some $i, p$. Let $b \in \mathcal{O}^{Z}$ and $e$ be such that $B=W_{e}^{H_{b}^{Z}}$. Then $T_{\langle 2(b+O(1))+1\rangle}=\operatorname{Decorate}\left(P_{b+O(1)}, h\right)$, where $\left|P_{b+O(1)}\right|=$ $\left|S_{b}\right| \cap\left|E_{b}\right|$. Therefore, there is some $\sigma \in T$ such that $T_{\sigma}=\operatorname{Decorate}\left(E_{b}, h\right)$. Since $E_{b}$ has a union at the root, this $\sigma$ is a union. Let $p=0^{e} 1$. We claim that $D_{\ell, p} \subseteq B$, where $D_{\ell}=\cup_{m} U_{\sigma\urcorner m} \cup V_{\sigma}$. Let $q$ be such that $p^{\frown} q \in D_{\ell}$. To finish the proof, we need to show that $[q] \subseteq B$.

For the remainder of this proof, any $X$ which meets the following conditions will be called sufficiently generic:

- $X \in \cap_{i} D_{i}$, and

- $X$ is 1 -generic relative to $H_{b+O(1)}^{Z}$

Observe that for every $r \in 2^{<\omega}$, there is a sufficiently generic $X \in M$ with $r \prec X$. Also, observe that for all such $X$ and all codes $R$ which are $c$-ranked for some $c \leq_{*} b+O(1)$, the second condition implies that $c, X$ and $R$ satisfy the conditions of Lemma 5.4. and so $X \in|\operatorname{Decorate}(R, h)|$ if and only if $X \in|R|$. Finally, by 
Proposition 3.9 for all sufficiently generic $X$ and all $\tau \in T$, we have $X \in\left|T_{\tau}\right|$ if and only if $X \in U_{\tau}$.

If $X$ is sufficiently generic and $p^{\prec} q \prec X$, then $X \in p^{\wedge} B$, and so $X \in\left|E_{b}\right|$, and so by Lemma 5.4 $X \in\left|\operatorname{Decorate}\left(E_{b}, h\right)\right|=\left|T_{\sigma}\right|$. Therefore, it is impossible that $X \in V_{\sigma}$, so we conclude $p^{\curlyvee} q \in U_{\sigma^{\frown} m}$ for some $m$. Therefore, for sufficiently generic $X$ with $p^{\frown} q \prec X$, we have $X \in\left|T_{\sigma \frown m}\right|$.

If $m=2 c+1$ for some $c \leq_{*} b+O(1)$, then $T_{\sigma^{\wedge} m}=\operatorname{Decorate}\left(P_{c}, h\right)$. But for any sufficiently generic $X$, we have $X \notin\left|P_{c}\right|$, so this case is impossible. Therefore, $m=2 n$ for some $\langle n\rangle \in E_{b}$. It follows from the definition of Decorate that $T_{\sigma^{\wedge} m}=$ Decorate $\left(\left(E_{b}\right)_{\langle n\rangle}, h\right)$. So for sufficiently generic $X$ with $p^{\prec} q \prec X$, we have $X \in$ $\left|\left(E_{b}\right)_{\langle n\rangle}\right|$.

Now we will use a property of the codes $E_{b}$ which follows from how they are defined at the beginning of the proof of Theorem 6.1. The code $E_{b}$ was obtained as the union of many codes $F_{b, e, r}$, at whose leaves the only options are $\left[0^{e} 1^{\frown} r\right]$ or $\emptyset$. The code $E_{b}$ was also post-processed so that it would be alternating, but while this process can break up the first-level subtrees $F_{b, e, r}$, it can never combine them together. (See the discussion at the end of Section 2.4 for details.) Therefore, for every $\langle n\rangle \in E_{b}$, there is an $r$ such that whenever $\langle n\rangle^{\wedge} \tau \in E_{b}$ is a leaf, its attached clopen set is either $\left[0^{e} 1^{\frown} r\right]$ or $\emptyset$. Fixing $r$ associated to $n=m / 2$ for the $m$ found above, we observe that an evaluation map on $\left(E_{b}\right)_{\langle n\rangle}$ that works for one $Y \in\left[0^{e} 1^{\frown} r\right]$ works for all such $Y$, and we conclude that $\left|\left(E_{b}\right)_{\langle n\rangle}\right|$ is equal to either $\emptyset$ or $\left[0^{e} 1^{\frown} r\right]$. It must be the latter because $X \in\left|\left(E_{b}\right)_{\langle n\rangle}\right|$ for all sufficiently generic $X$ with $p^{\frown} q \prec X$. It follows that $[r] \subseteq B$. Furthermore, any sufficiently generic $X$ that does not extend $p^{\wedge} r$ must be out of $\left|\left(E_{b}\right)_{\langle n\rangle}\right|$, so it must be that $[q] \subseteq[r]$. Therefore, $[q] \subseteq B$, as desired.

\section{Application to the Borel dual Ramsey theorem}

As an application of Theorem 4.3, we identify a natural formulation of the Borel dual Ramsey theorem for 3 partitions and $\ell$ colors (Borel-DRT ${ }_{\ell}^{3}$ ) as a principle which lies strictly below $\mathrm{ATR}_{0}$, but all of whose $\omega$-models are closed under hyperarithmetic reduction.

Theorem 8.1 (Borel dual Ramsey theorem, CS84]). For every Borel $\ell$-coloring of the set of partitions of $\omega$ into exactly $k$ pieces, there is an infinite partition $p$ of $\omega$ and a color $i<\ell$ such that every way of coarsening $p$ down to exactly $k$ pieces is given color $i$.

Since the set of partitions of $\omega$ into exactly $k$ pieces can be coded naturally as a Borel subset of $k^{\omega}$, a natural way to formulate the hypotheses of the above theorem is roughly "Whenever there are Borel codes $T_{1}, \ldots T_{\ell}$ such that for every $X \in k^{\omega}$, we have $X \in\left|\cup_{i<\ell} T_{i}\right|, \ldots$ " (See below for a precise formalization).

Therefore, the Borel dual Ramsey theorem has a natural formulation in terms of completely determined Borel sets. In PV85, DFSW17, it was shown that a solution to Borel-DRT $\mathrm{D}_{\ell}^{k}$ can in general be obtained by a two-step process:

(1) Use the fact that every Borel set has the property of Baire to come up with a Baire approximation to each color in the given coloring.

(2) Apply a purely combinatorial principle $\mathrm{CDRT}_{\ell}^{k}$ to a coloring of $(k-1)^{<\omega}$ obtainable from the Baire approximation from (1). 
If we represent the coloring in the natural way described below, then CD-PB can be used to carry out (1). It was known to Simpson (see [DFSW17]) that $\mathrm{CDRT}_{\ell}^{3}$ follows from Hindman's Theorem (HT), which follows from $\mathrm{ACA}_{0}^{+}$by BHS87. Therefore, the following natural formalization of Borel-DRT ${ }_{\ell}^{3}$ follows from CD-PB $+\mathrm{ACA}_{0}^{+}$. We first give the formalization of the space of $k$-partitions of $\omega$, and then the formalization of Borel-DRT ${ }_{\ell}^{3}$.

Definition 8.2 (Partitions of $\omega$, [DFSW17]). In $\mathrm{RCA}_{0}$, a partition of $\omega$ into exactly $k$ pieces is a function $p \in k^{\omega}$ such that $p$ is surjective, and for each $i<k-1$,

$$
\min \{n: p(n)=i\}<\min \{n: p(n)=i+1\} .
$$

A partition of $\omega$ into infinitely many pieces is a surjective function $p \in \omega^{\omega}$ which satisfies the above condition for each $i \in \omega$.

The set of partitions described above is an open subset of $k^{\omega}$ representable in $\mathrm{RCA}_{0}$ by a completely determined Borel code, as the reader can verify. (For the case $k=3$, the set in question is the union of the sets $O_{a, b}$ introduced at the start of the proof of Theorem 8.5.) Let $P_{3}$ denote this completely determined Borel code in the case $k=3$.

Definition 8.3 (Formal Borel dual Ramsey theorem for 3 partitions and $\ell$ colors). In $\mathrm{RCA}_{0}$, Borel-DRT $T_{\ell}^{3}$ is the principle which states: Whenever $T_{0}, \ldots T_{\ell-1}$ are Borel codes such that for all $X \in\left|P_{3}\right|$, we have $X \in\left|\bigcup_{i<\ell} T_{i}\right|$, then there is an infinite partition $p$ of $\omega$ and a color $i<\ell$ such that whenever $X \in\left|P_{3}\right|, X \circ p \in\left|T_{i}\right|$.

We would like to say the hypotheses of the theorem imply that the $\left\{T_{i}\right\}_{i<\ell}$ are all completely determined. This is not quite true (perhaps $X \in\left|T_{i}\right|$ is not completely determined for some $\left.X \notin\left|P_{3}\right|\right)$. However, a small modification of the existing codes makes them completely determined.

Lemma 8.4. $\left(\mathrm{ACA}_{0}\right)$ Suppose that $S$ is a completely determined Borel code and $T$ is a Borel code. Suppose that for all $X \in|S|$, there is an evaluation map for $X$ in $T$. Then there is a completely determined Borel code $R$ such that for all $X \in|S|$, we have

$$
X \in|T| \Longleftrightarrow X \in|R| .
$$

Proof. Let $R$ be obtained from $T$ by replacing each leaf $\sigma$ in $T$ with the intersection of $S$ and the clopen set coded by $\ell(\sigma)$ in $T$. If $X \in|S|$, then an evaluation map for $X$ in $R$ is obtained by starting with an evaluation map for $X$ in $T$ and then filling in the evaluation map for $X \in|S|$ at all the places where $S$ appears in $R$. If $X \in\left|S^{c}\right|$, an evaluation map for $X$ in $R$ is obtained by filling in all the original nodes of $T$ with 0 , filling in the evaluation map for $X$ in $S$ at all the places where $S$ appears in $R$, and filling in the correct values on the remaining leaves which were copied from $T$.

(Note: it does not work to let $R$ be simply the intersection of $S$ and $T$, because the definition of completely determined requires that the entire evaluation map be filled out, even if most of it is not used.)

It follows that if $\left(T_{i}\right)_{i<k}$ satisfy the hypotheses of the formal Borel dual Ramsey theorem above, they can be taken to be completely determined without loss of generality. Therefore, the discussion preceding the formal definitions proves that CD-PB + ACA $_{0}^{+} \vdash$ Borel-DRT ${ }_{\ell}^{3}$ over RCA . 
The $\omega$-model which was constructed to prove Theorem 4.3 is closed under hyperarithmetic reduction, and therefore satisfies $\mathrm{ACA}_{0}^{+}$as well as CD-PB. Therefore, Borel-DRT $T_{\ell}^{3}$ holds in this model, while $\mathrm{ATR}_{0}$ does not. This shows that the formulation of Borel-DRT $T_{\ell}^{3}$ discussed here is strictly weaker than $\mathrm{ATR}_{0}$.

On the other hand, we have the following, which essentially follows from a more detailed version of the analysis in Section 4 of [DFSW17.

Theorem 8.5. Let $\ell \in \omega$ with $\ell \geq 2$. Every $\omega$-model of Borel-DRT $T_{\ell}^{3}$ is closed under hyperarithmetic reduction.

Proof. It suffices to consider the case $\ell=2$. We will first define some important subsets of $3^{\omega}$. For each $a, b$ with $0<a<b$, let $O_{a, b}$ be the clopen set given by the finite collection of strings

$$
O_{a, b}=\left\{\sigma \in 3^{b+1}: a=\min \{n: \sigma(n)=1\} \text { and } b=\min \{n: \sigma(n)=2\}\right\}
$$

Then the set of partitions of $\omega$ into exactly 3 pieces is given by $P_{3}=\bigcup_{0<a<b} O_{a, b}$.

Let $M$ be the second-order part of an $\omega$-model $\mathcal{M}$ of Borel-DRT ${ }_{2}^{3}$. We first show that $\mathcal{M}$ satisfies $\mathrm{ACA}_{0}$. Let $A \in M$. Let $R$ be the following labeled Borel code 4

$$
R=\bigcup_{0<a<b} \bigcap_{s>b} C_{a, b, s} \text { where } C_{a, b, s}= \begin{cases}O_{a, b} & \text { if } A_{b}^{\prime}\left\lceil a=A_{s}^{\prime}\lceil a\right. \\ \emptyset & \text { otherwise. }\end{cases}
$$

Then $R$ is completely determined. For any $X \in 3^{\omega}$, there is at most one pair $a, b$ such that $X \in O_{a, b}$, so an evaluation map for $X$ in $R$ may safely put zeros at every node of $R$ except for the root and the nodes of the distinguished subtree $\cap_{s>b} C_{a, b, s}$. The leaves of that subtree can be $X \oplus A$-computably filled out. Then the root of $R$ and the root of the subtree $\cap_{s>b} C_{a, b, s}$ may be non-uniformly supplied with their unique correct values.

Exactly as in the proof of [DFSW17, Theorem 4.5], we now show that for any infinite partition $p$ of $\omega$ which is homogeneous for the coloring defined by $|R|,\left|R^{c}\right|$, the principal function of $p$ dominates the least modulus function for $A^{\prime}$. For each $i$, let $p_{i}=\min \{n: p(n)=i\}$ (these are the minimum elements of the blocks of $p$ ). First we claim that $p$ is homogeneous for color $R$. Let $s$ be large enough that $A_{s}^{\prime}\left\lceil p_{1}=A^{\prime}\left\lceil p_{1}\right.\right.$. Let $j$ be large enough that $p_{j}>s$. Then the coarsening of $p$ which keeps blocks 1 and $j$, while collapsing all other blocks in with the zero block, is an element of $R$. By similar reasoning, but now looking at the 3-partition of $\omega$ obtained from $p$ by keeping the only the $i$ and $(i+1)$ blocks separate from the 0 block, we have $A_{p_{i+1}}^{\prime}\left\lceil p_{i}=A^{\prime}\left\lceil p_{i}\right.\right.$. Thus $p \geq_{T} A^{\prime}$. Therefore, $\mathcal{M} \models \mathrm{ACA}_{0}$.

Now suppose that $A \in M$ and $3 \cdot 5^{e} \in \mathcal{O}^{A}$. Suppose that for all $d \leq_{\mathcal{O}} 3 \cdot 5^{e}$, we have $H_{d}^{A} \in M$. Then we claim that $H_{3 \cdot 5^{e}}^{A} \in M$. By a result of Jockusch Joc68 discussed in more detail below, the hyperarithmetic sets are exactly those that can be computed from sufficiently fast-growing functions. As in DFSW17, Theorem 4.7], we construct a Borel coloring which forces any solution to Borel-DRT ${ }_{2}^{3}$ to compute a sufficiently fast-growing function. To prove the associated Borel code is completely determined, we need a more detailed analysis than what was given in DFSW17.

\footnotetext{
${ }^{4}$ We use standard computability-theoretic notation: for any $s \in \mathbb{N}$, let $A_{s}^{\prime}$ denote $\{x<s$ : $\left.\Phi_{x, s}^{A}(x) \downarrow\right\}$, and for any $X$ let $X\lceil s$ denote the string $\sigma$ of length $s$ describing the characteristic function of $X$ on $\{0, \ldots, s-1\}$.
} 
More specifically, Jockusch's result has plenty of uniformity: there are computable functions $h$ and $k$ such that for all $d \in \mathcal{O}^{A}$, whenever $g: \omega \rightarrow \omega$ dominates the increasing function

we have

$$
f_{d}(n):=\Phi_{h(d)}^{H_{d}^{A}}(n)
$$

$$
\Phi_{k(d)}(A \oplus g)=H_{d}^{A} .
$$

(To get this from the proof of [Joc68, Theorem 6.8], apply [Rog87, Exercise 16-98] to conclude that the sets $H_{d}^{A}$ are in fact uniformly Turing equivalent to implicitly $\Pi_{1}^{0}(A)$-definable functions $f_{d}$.)

Uniformly in $d \in \mathcal{O}^{A}$ and $a, b, \in \omega$, and $A$, there are Borel codes $C_{a, b, d}$ of wellfounded rank $d+O(1)$ such that

$$
C_{a, b, d}= \begin{cases}O_{a, b} & \text { if } b \geq f_{d}(a) \\ \emptyset & \text { otherwise. }\end{cases}
$$

The uniformity follows from the existence of $h$ above and the $A$-uniformity of producing a formula of $L_{\omega_{1}, \omega}$ to assess facts about $H_{d}^{A}$ (Proposition 2.1).

For each $n<\omega$, let $d_{n}=\Phi_{e}(n)$. Now let $R$ be the labeled Borel code

$$
R=\bigcup_{0<a<b} \bigcap_{i \leq a} C_{a, b, d_{i}}
$$

For any $X \in 3^{\omega}$, there is at most one pair of $a, b$ such that $X \in O_{a, b}$, so as above, any evaluation map for $X$ in $R$ can safely fill in zeros everywhere except for the root of $R$ and the distinguished subtree rooted at $\cap_{i \leq a} C_{a, b, d_{i}}$. This subtree has wellfounded rank $d_{a}+O(1)$, so the unique evaluation map on it is $H_{d_{a}+O(1)}^{A}$-computable. Because $H_{d}^{A} \in M$ for all $d \leq_{\mathcal{O}} 3 \cdot 5^{e}$, this evaluation map exists in $M$. Therefore, $R$ is completely determined in $M$.

Now let $p \in M$ be any infinite partition of $\omega$ which is a solution to Borel-DRT ${ }_{2}^{3}$ for the coloring $|R|,\left|R^{c}\right|$. Define, for each $i$,

$$
p_{i}=\min \{n: p(n)=i\} .
$$

Continuing to copy the proof of [DFSW17, Theorem 4.5], for every $0<s<t$, consider the coarsening $X_{s, t}$ of $p$ obtained by keeping the $s$ and $t$ blocks of $p$ and collapsing all other blocks to 0 . Since $t$ can be chosen arbitrarily large, for every $s$ there is a $t$ such that

$$
X_{s, t} \in \bigcap_{i \leq p_{s}} C_{p_{s}, p_{t}, d_{i}}
$$

and therefore $P_{3} \circ p$ is monochromatic for color $R$, and $s<t$ implies that for all $i \leq p_{s}$, we have $p_{t} \geq f_{d_{i}}\left(p_{s}\right)$. Therefore, $p$ computes a sequence of functions $\left\{g_{i}: i \in \omega\right\}$ such that for all $i$ and $n, g_{i}(n) \geq f_{d_{i}}(n)$. (Given $i$ and $n$, let $s$ be large enough that $i, n \leq p_{s}$, and output $p_{s+1}$.) Therefore, $A \oplus p$ computes

$$
\bigoplus_{i} \Phi_{k\left(d_{i}\right)}\left(A \oplus g_{i}\right)=\bigoplus_{i} H_{d_{i}}^{A}=H_{3 \cdot 5^{e}}^{A}
$$

as was needed.

We end this section with a question about robustness. The formalization of Borel-DRT ${ }_{2}^{3}$ given above is one we find quite natural. However, another possible way to state the hypothesis of this theorem would be "Whenever there are Borel codes $T_{1}, \ldots T_{\ell}$ such that for every $X \in k^{\omega}$, there is an $i$ such that $X \in\left|T_{i}\right|, \ldots$ " 
The subtle difference lies in the fact that if $X \in\left|\cup_{i<\ell} T_{i}\right|$, the evaluation map for $X$ in that code must also prove that $X \in\left|T_{i}\right|$ or $X \in\left|T_{i}^{c}\right|$ for each $i<\ell$. In the slight variant just mentioned, it is enough to know that for some $i, X \in T_{i}$ (and possibly have no information about $X$ in the codes $T_{j}$ for $j \neq i$.) This variant does not, at least on its face, lead to any conclusion about whether, or in what sense, any of the $T_{i}$ must be completely determined.

Question 8.6. How robust is the given formalization of Borel-DRT ${ }_{2}^{3}$ ? In particular, is it equivalent to the variant described above?

\section{Questions}

Several directions of further questions immediately suggest themselves. Most results here concern $\omega$-models. It is not immediately clear how to formalize the statement "for every $Z$, there is a $\Delta_{1}^{1}(Z)$-generic" in reverse mathematics. Once a reasonable reverse mathematics way of formalizing these principles is established, it would be natural to ask how these principles are related to principles about (completely determined) Borel sets.

In the context of $\omega$-models, there are some gaps remaining. For example, we have seen that every $\omega$-model of CD-PB models $L_{\omega_{1}, \omega}$-CA and the existence of $\Delta_{1}^{1}$ generics.

Question 9.1. Suppose $M \subseteq 2^{\omega}$ is closed under join, satisfies $\mathrm{L}_{\omega_{1}, \omega}-\mathrm{CA}$, and for every $Z \in M$, there is a $G \in M$ that is $\Delta_{1}^{1}(Z)$-generic. Does it follow that $\mathcal{M} \models$ CD-PB?

One way that the above question could have a negative answer would be if CD-PB implied some theory of hyperarithmetic analysis strictly stronger than $\mathrm{L}_{\omega_{1}, \omega}-\mathrm{CA}$.

Question 9.2. Which theorems of hyperarithmetic analysis are implied by CD-PB, and which are incomparable with it?

We built an $\omega$-model of CD-PB by adjoining many mutually $\Sigma_{1}^{1}$-generics.

Question 9.3. Does every $\omega$-model of CD-PB contain a $\Sigma_{1}^{1}$-generic?

Whether in $\omega$-models or full reverse mathematics, many other theorems involving Borel sets may now have interesting reverse mathematics content when considering their completely determined versions. We leave the similar analysis of "Every completely determined Borel set is measurable" to future work. We mention that the statement "Every completely determined Borel set has the perfect set property" is equivalent to $\mathrm{ATR}_{0}$, because "Every closed set has the perfect set property" already implies $A_{T R}$ by [Sim09, V.5.5], so here the way of defining a Borel set does not add additional strength.

Turning now to Borel-DRT ${ }_{\ell}^{3}$, we have seen that any $\omega$-model of it is closed under hyperarithmetic reduction.

Question 9.4. Is Borel-DRT ${ }_{\ell}^{3}$ a theory of hyperarithmetic analysis?

For any instance of Borel-DRT ${ }_{\ell}^{3}$ that is truly well-founded, there is a solution hyperarithmetic in the instance. However, we do not know anything about the complexity of solutions to non-standard instances of Borel-DRT ${ }_{\ell}^{3}$. In particular, we do not know if Borel-DRT ${ }_{\ell}^{3}$ holds in $H Y P$. 
Finally, there is the issue of robustness. There are some possible variations on what could be considered as an evaluation map. For example, a weaker version of an evaluation map would be a partial function $f: \subseteq T \rightarrow\{0,1\}$ such that $f(\lambda)$ is defined; and whenever $\sigma \in T$ is a $\cup$, and $f(\sigma)=1$, there is an $n$ such that $f\left(\sigma^{\frown} n\right)=1$; and whenever $\sigma \in T$ is a $\cap$ and $f(\sigma)=1$, for all $n, \sigma^{\frown} n \in T$ implies $f\left(\sigma^{\frown} n\right)=1$; and similarly for when $f(\sigma)=0$. Such a partial function has a natural interpretation as a winning strategy in the game in which one player tries to prove that a real is in the given Borel set while another player tries to prove that it is out. We have used the longer name "completely determined Borel set" for our notion in order to reserve the term "determined Borel set" for this variant. We did not investigate, but it would be interesting to know, the extent to which the results of this paper are robust under this and other variations on when we consider Borel set to be well-defined in reverse mathematics.

\section{REFERENCES}

[BHS87] Andreas R. Blass, Jeffry L. Hirst, and Stephen G. Simpson. Logical analysis of some theorems of combinatorics and topological dynamics. In Logic and combinatorics (Arcata, Calif., 1985), volume 65 of Contemp. Math., pages 125-156. Amer. Math. Soc., Providence, RI, 1987.

[Con12] Chris J. Conidis. Comparing theorems of hyperarithmetic analysis with the arithmetic Bolzano-Weierstrass theorem. Trans. Amer. Math. Soc., 364(9):4465-4494, 2012.

[CS84] Timothy J. Carlson and Stephen G. Simpson. A dual form of Ramsey's theorem. Adv. in Math., 53(3):265-290, 1984.

[DFSW17] Damir Dzhafarov, Stephen Flood, Reed Solomon, and Linda Brown Westrick. Effectiveness for the Dual Ramsey Theorem. Submitted 2017. Available arXiv:1710.00070.

[DH10] Rodney G. Downey and Denis R. Hirschfeldt. Algorithmic Randomness and Complexity. Theory and Applications of Computability. Springer, New York, 2010.

[Fri75] Harvey Friedman. Some systems of second order arithmetic and their use. Proceedings of the International Congress of Mathematicians (Vancouver, B. C., 1974), Vol. 1, pages 235-242, 1975.

[GM17] Noam Greenberg and Benoit Monin. Higher randomness and genericity. Forum Math. Sigma, 5:e31, 41, 2017.

[Har68] Joseph Harrison. Recursive pseudo-well-orderings. Trans. Amer. Math. Soc., 131:526$543,1968$.

[Joc68] Carl G. Jockusch, Jr. Uniformly introreducible sets. J. Symbolic Logic, 33:521-536, 1968.

[Mon06] Antonio Montalbán. Indecomposable linear orderings and hyperarithmetic analysis. $J$. Math. Log., 6(1):89-120, 2006.

[Mon09] Antonio Montalbán. Theories of hyperarithmetic analysis. Slides from talk at the conference in honor of the 60th birthday of Harvey Friedman, 2009.

[PV85] Hans Jürgen Prömel and Bernd Voigt. Baire sets of $k$-parameter words are Ramsey. Trans. Amer. Math. Soc., 291(1):189-201, 1985.

[Rog87] Hartley Rogers, Jr. Theory of Recursive Functions and Effective Computability. MIT Press, Cambridge, MA, second edition, 1987.

[Sac90] Gerald E. Sacks. Higher Recursion Theory. Perspectives in Mathematical Logic. Springer-Verlag, Berlin, 1990.

[Sim09] Stephen G. Simpson. Subsystems of Second Order Arithmetic. Perspectives in Logic. Cambridge University Press, Cambridge; Association for Symbolic Logic, Poughkeepsie, NY, second edition, 2009.

[Ste78] John R. Steel. Forcing with tagged trees. Ann. Math. Logic, 15(1):55-74, 1978.

[VW77] Robert Alan Van Wesep. Subsystems of second-order arithmetic, and descriptive set theory under the axiom of determinateness. ProQuest LLC, Ann Arbor, MI, 1977. Thesis (Ph.D.)-University of California, Berkeley.

[Yu06] Liang Yu. Lowness for genericity. Arch. Math. Logic, 45(2):233-238, 2006. 
Google LlC, 111 8Th Ave., New York, NY 10011, U.S.A.

E-mail address: eric.astor@gmail.com

Department of Mathematics, University of Connecticut, Storrs, Connecticut U.S.A.

E-mail address: damir.dzhafarov@uconn.edu

Department of Mathematics, University California-Berkeley, Berkeley, California U.S.A.

E-mail address: antonio@math.berkeley.edu

Department of Mathematics, University of Connecticut, Storrs, Connecticut U.S.A.

E-mail address: solomon@math.uconn.edu

Department of Mathematics, Penn State University, University Park, Pennsylvania U.S.A.

E-mail address: westrick@psu.edu 